\title{
Mutant p53 establishes targetable tumor dependency by promoting unscheduled replication
}

\author{
Shilpa Singh, ${ }^{1}$ Catherine A. Vaughan, ${ }^{1}$ Rebecca A. Frum, ${ }^{2}$ Steven R. Grossman, ${ }^{2}$ Sumitra Deb, ${ }^{1}$ and Swati Palit Deb ${ }^{1}$ \\ 'Department of Biochemistry and Molecular Biology, ²Department of Internal Medicine, Division of Hematology, Oncology and Palliative Care, VCU Massey Cancer Center, Virginia Commonwealth University, \\ Richmond, Virginia, USA.
}

\begin{abstract}
Gain-of-function (GOF) p53 mutations are observed frequently in most intractable human cancers and establish dependency for tumor maintenance and progression. While some of the genes induced by GOF p53 have been implicated in more rapid cell proliferation compared with p53-null cancer cells, the mechanism for dependency of tumor growth on mutant p53 is unknown. This report reveals a therapeutically targetable mechanism for GOF p53 dependency. We have shown that GOF p53 increases DNA replication origin firing, stabilizes replication forks, and promotes micronuclei formation, thus facilitating the proliferation of cells with genomic abnormalities. In contrast, absence or depletion of COF p53 leads to decreased origin firing and a higher frequency of fork collapse in isogenic cells, explaining their poorer proliferation rate. Following genomewide analyses utilizing ChIP-Seq and RNA-Seq, GOF p53-induced origin firing, micronuclei formation, and fork protection were traced to the ability of GOF p53 to transactivate cyclin A and CHK1. Highlighting the therapeutic potential of CHK1's role in COF p53 dependency, experiments in cell culture and mouse xenografts demonstrated that inhibition of CHK1 selectively blocked proliferation of cells and tumors expressing COF p53. Our data suggest the possibility that checkpoint inhibitors could efficiently and selectively target cancers expressing GOF p53 alleles.
\end{abstract}

\section{Introduction}

P53 is among the most commonly mutated genes in various cancers, but particularly in lung cancer $(1,2)$. The majority of p53 mutations found in human cancers, including lung cancers, are missense mutations that have "driver" roles $(3,4)$, suggesting a selective advantage for retaining the mutated allele. It is well established that loss of WT p53 increases vulnerability to tumor formation (5), whereas tumor-derived mutants of p53 show gain-of-function (GOF) properties, which confer a selective growth advantage to cancer cells. Several mouse models have been reported to investigate GOF properties of p53 mutants (6-10). In addition to loss of WT p53 function, the ability of GOF p53 to activate transcription of proliferative genes (11-13) or to deregulate signaling pathways (14) has been connected to its oncogenic properties. Inhibition of tumor formation by knockdown of endogenous mutant p53 has been demonstrated in human lung cancer cells using RNAi and knockin (KI) mouse models $(15,16)$. A recent study has reported that inactivation or destabilization of GOF p53 reduces tumor growth in mice, extending their survival (17). These observations demonstrate a dependence of the tumor-formation ability of cancer cells on GOF p53, a phenomenon described as oncogene addiction (18). The selective growth advantage of cancer cells harboring GOF p53 mutation and the requirement for the continued expression of GOF p53 mutants to maintain tumor growth therefore argue

Conflict of interest: The authors have declared that no conflict of interest exists. Submitted: March 24, 2016; Accepted: February 16, 2017.

Reference information: J Clin Invest. 2017;127(5):1839-1855.

https://doi.org/10.1172/JCl87724. that cancer cells expressing GOF p53 alleles are indeed dependent on GOF 553 protein, which therefore can be targeted therapeutically in cancer.

How GOF p53 induces oncogenic cell proliferation or why the proliferation of cancer cells might be addicted to GOF p53 is unknown. Loss of WT 553 and expression of GOF p53 are both known to deregulate the cell cycle and to induce untimely $\mathrm{S}$ phase entry (5), yet GOF p53 also specifically confers a selective proliferation advantage. To determine the mechanism of GOF p53-dependent growth of cancer cells, we investigated the architecture of genome duplication in the presence and absence of GOF p53. Since GOF p53 mutation is prevalent in lung cancer, human lung cancer or primary mouse lung cells were used for these experiments. Our data indicate that, in comparison with p53-null, p53-depleted, or loss-of-function (non-GOF) p53-expressing cells, lung cells with GOF p53 show a higher frequency of origin firing at early $\mathrm{S}$ phase, promoting rapid genome duplication with errors, as demonstrated by early entry into mitosis and increase in micronuclei formation. Consistent with its increased origin-firing activity, GOF p53 increased expression of the intra-S phase checkpoint kinase CHK1, known to prevent collapse of replication forks. Thus, in comparison with $p 53^{--}$cells, cells with GOF p53 show higher levels of CHK1 and phosphorylated CHK1 and reduced frequency of replication fork collapse. In contrast, $p 53^{--}$or p53-depleted cells show decreased origin firing, higher frequency of replication fork collapse, and increased levels of chromatinassociated histone $\mathrm{H} 2 \mathrm{AX}(\gamma \mathrm{H} 2 \mathrm{AX})$. Compromise of GOF p53-mediated transcriptional activation abrogated its ability to increase origin firing, form micronuclei, and activate the intra-S phase checkpoint, reestablishing replication fork collapse and reduced cell proliferation. Genome-wide analyses revealed that GOF p53 recognizes the 
A

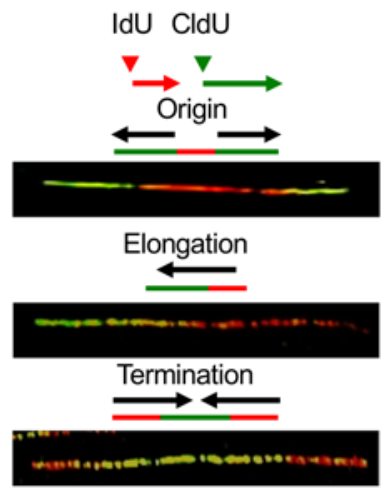

B

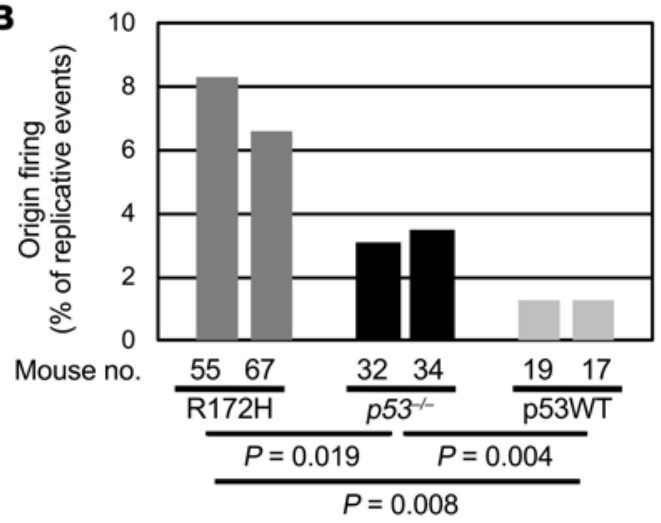

C

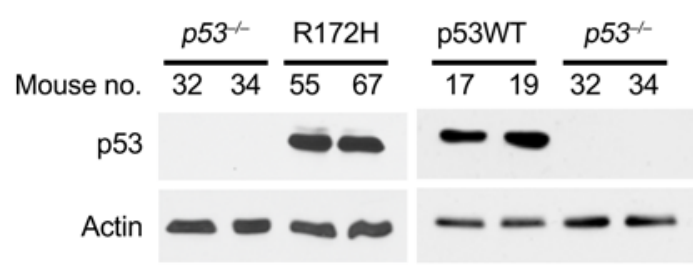

D

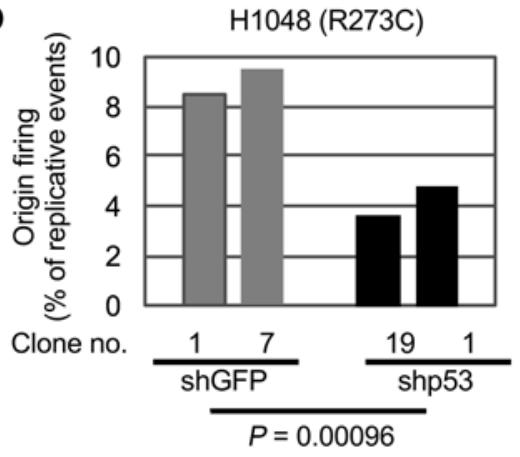

E

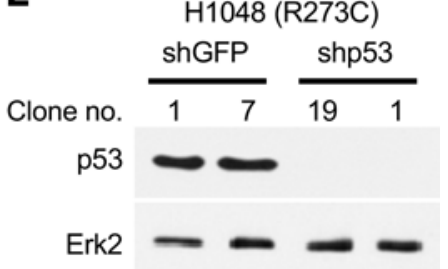

$\mathbf{F}$

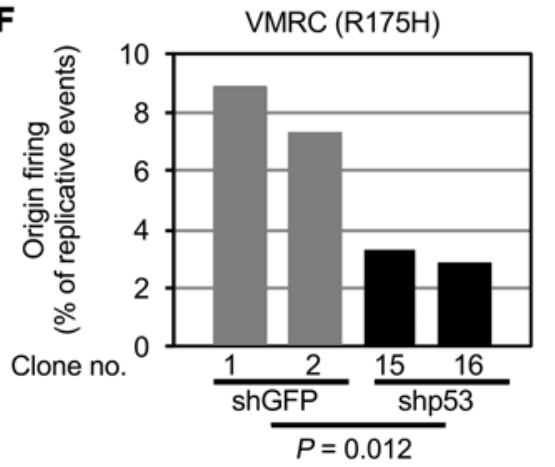

G Clone no. $\frac{\text { shGFP }}{12} \frac{\text { shp53 }}{15 \quad 16}$ p53

Actin VMRC (R175H)

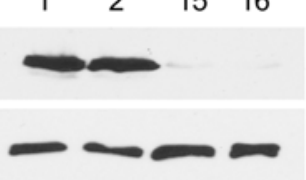

Figure 1. Compared with p53-null or p53-depleted cells, transformed or nontransformed lung cells harboring G0F p53 fire more DNA replication origins at early $\mathbf{S}$ phase. Fiber images $(\mathbf{A})$ display bidirectional origin and elongating or terminating forks. Percentages of origins in replicating fibers from lung cells of $p 53^{-1-}, \mathrm{p} 53 \mathrm{R} 172 \mathrm{H}-\mathrm{KI}(\mathrm{R} 172 \mathrm{H})$, and WT (p53WT) mice (B) and mock-depleted (shCFP) or p53-depleted (shp53) lung cancer cell lines H1048 (D), which harbors R273C, and VMRC, which harbors R175H (F), are shown by bar graphs. Expression of p53 was determined by immunoblot analysis in each experiment (C, E, G). WT p53 expression (C) was visualized by analyzing higher protein (6-fold over p53R172HKI lung cell extract) and longer exposure. Actin is a loading control. Lung cells from 2 mice of each construct or 2 clones stably expressing shGFP or shp53 were analyzed. The $P$ values calculated using Student's $t$ test are shown at the bottom of the bar graphs. Additional fiber images are shown in Supplemental Figures 2 and 3 . Each experiment was repeated 3 times, and representative data are shown.

promoters of genes encoding cyclin A (CCNA2), needed for origin firing (19-21), and CHK1, needed for preventing collapse of replication forks (22-24), and activates their expression in a cell cycledependent manner at the level of transcription.

Collapse of replication forks is known to induce either cell death or gross chromosomal rearrangement due to incomplete DNA replication $(25,26)$. Consistently, experiments utilizing a mouse xenograft model revealed that inhibition of $\mathrm{CHK} 1$ reduced the size of tumors generated from a human lung cancer cell line with GOF p53 mutation more efficiently than GOF p53 knockdown cells and selectively reduced proliferation of cultured lung cancer cells with GOF p53 mutation. These data provide compelling evidence that GOF p53 establishes oncogene addiction in cancer cells by generating and fostering DNA replication forks and that inactivation of these functions of GOF p53 may represent a promising therapeutic avenue in cancer.

\section{Results}

GOF $p 53$ mutation and loss of WT $p 53$ hasten time of S phase entry to a similar extent. While loss of $\mathrm{p} 53$ deregulates the cell cycle to enter $S$ phase (5), several laboratories have demonstrated that GOF p53 accelerates cell proliferation and tumor growth in comparison 
A
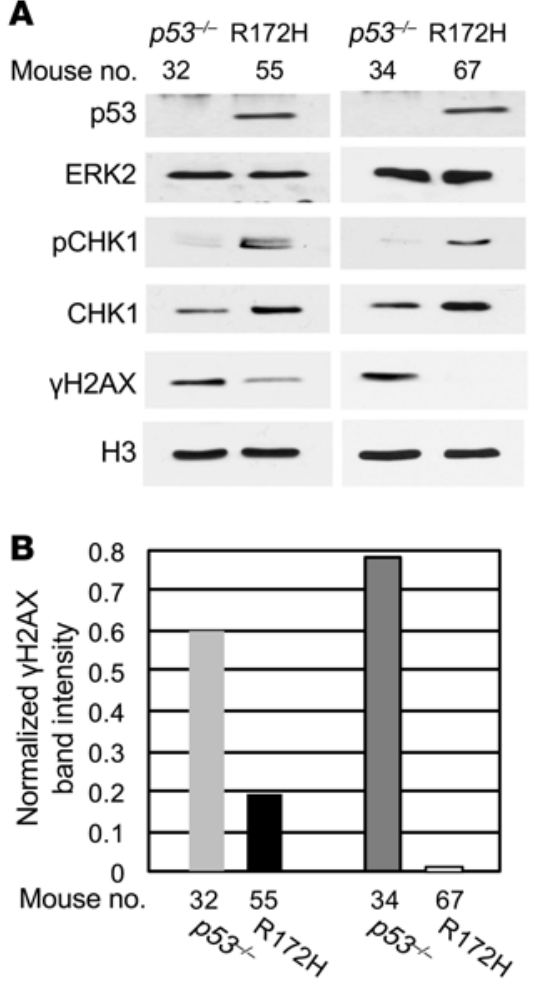

E

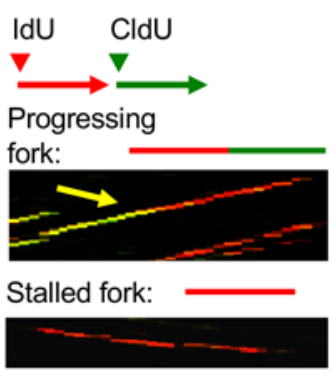

$\mathbf{F}$

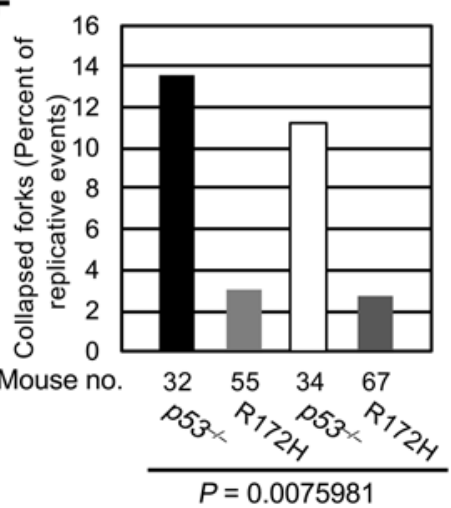

C

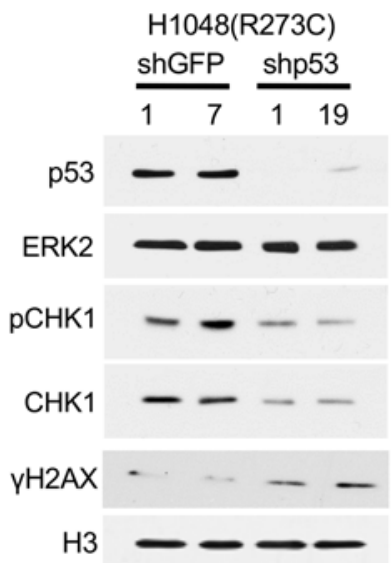

D

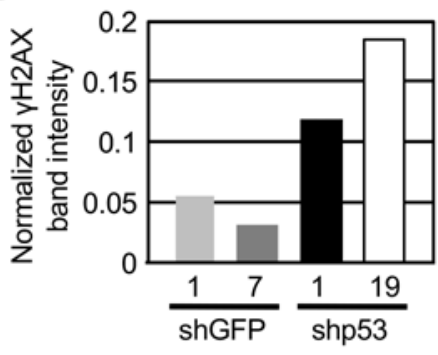

G
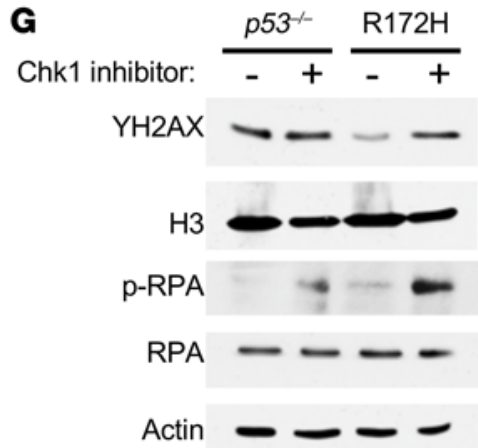

H

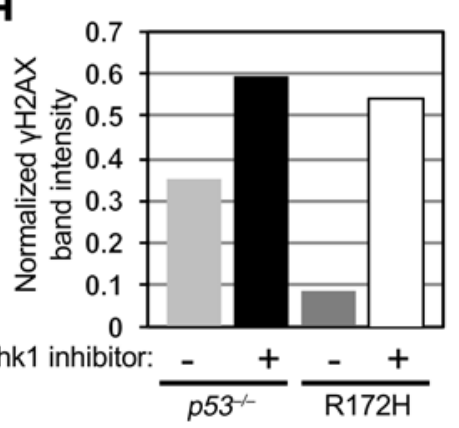

Figure 2. GOF p53 activates intra-S phase checkpoint, preventing collapse of replication forks. Detection of $\mathrm{p}-\mathrm{CHK} 1$, CHK1, and chromatin-associated $\gamma \mathrm{H} 2 \mathrm{AX}$ by immunoblot analysis using (A) lung cells of $p 53^{-/-}$and $p 53 \mathrm{R} 172 \mathrm{H}-\mathrm{KI}$ (R172H) mice and (C) mock-depleted (shGFP) or p53depleted (shp53) H1048 lung cancer cells. For detection of chromatin-associated $\gamma \mathrm{H} 2 \mathrm{AX}$, insoluble chromatin fraction was acid extracted and analyzed by immunoblotting. Band intensities of $\gamma \mathrm{H} 2 \mathrm{AX}$ normalized by $\mathrm{H} 3$ levels are shown by bar graphs (B and $\mathbf{D}$ ). Lung cells from 2 mice of each construct or 2 clones stably expressing shCFP or shp53 were analyzed. The experimental strategy for detection of replication fork collapse by fiber analysis is shown by a schematic (E). Collapse of progressing replication forks was assayed by fiber analysis of replicating DNA. The bar graph (F) compares the percentage of red-only tracks (indicated by arrows in the fiber images) in replicating fibers. Approximately 200 untangled fibers from each sample were scored. The $P$ value calculated using Student's $t$ test is indicated at the bottom of the bar graph (F). Lung cells from 2 mice of each construct were analyzed. Chromatin-associated $\gamma \mathrm{H} 2 \mathrm{AX}$ levels in lung cells from $p 53^{-/-}$and $\mathrm{R} 172 \mathrm{H}$ mice after treatment with a CHK1 inhibitor, PF477736, were determined by immunoblot analysis (G). $\gamma \mathrm{H} 2 \mathrm{AX}$ band intensities normalized by $\mathrm{H} 3$ levels are shown by bar graphs (H). p-RPA (Ser4/Ser8) was detected by immunoblot analysis (C) to ensure activity of PF477736. Each experiment was repeated 3 times, and representative data are shown.

to incorporate the nucleotide analogue iododeoxyuridine (IdU) was determined at various times after replating by immunostaining IdU and scoring IdU-labeled cells using confocal microscopy. The data showed that lung cells from p53-null and p53R172H-KI mice entered S phase at similar times, with $20 \%$ to $23 \%$ cells incorporating IdU at 12 hours after replating density-arrested cells, while lung cells from normal (WTp53) mice showed 5\% to 7\% IdU-labeled cells under similar conditions (Supplemental Figure 1; supplemental material available online with this article; https://doi.org/10.1172/ JCI87724DS1). These data suggest that the absence of $\mathrm{p} 53$ or presence of $\mathrm{p} 53 \mathrm{R} 172 \mathrm{H}$ hastens S phase entry to a similar extent.

Compared with p53-null cells, the frequency of DNA replication origin firing is higher in cells expressing GOF p53 mutants. Since GOF p53 mutants activate expression of genes required for DNA replication (27), the ability of these mutants to increase firing of DNA replication origins during $S$ phase entry was determined. To determine whether GOF p53 mutants alter frequency of DNA replication origin firing in otherwise normal cells, lung cells from $p 53^{--}$and p53R172H-KI mice were cultured and the frequency of origin firing during early $\mathrm{S}$ phase was with p53-null or -knockdown cells $(15,16)$ and upregulates expression of cell-proliferating genes (27). Therefore, experiments were carried out to determine whether GOF p53 hastens the time of S phase entry compared with p53-null cells. Short-term cultures of lung cells from p53R172H-KI (human R175H) and p53-null mice were partially synchronized by density arrest, and their ability determined using fiber analysis of replicating DNA using methods published earlier (28-30). Cells were partially synchronized by density arrest and replating and sequentially labeled with IdU and chlorodeoxyuridine (CldU) at early S phase. Cellular genomic DNA was then spread on slides, and replicating DNA fibers were detected by immunostaining of incorporated IdU and CldU using 

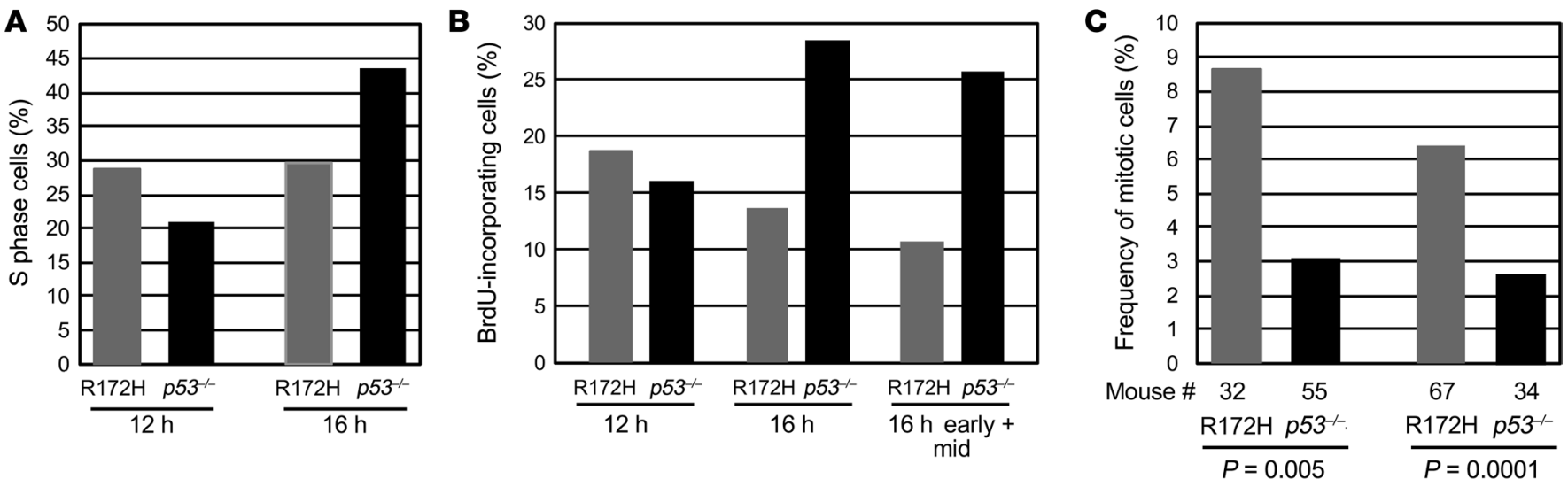

Figure 3. GOF p53 promotes rapid genome duplication and mitotic entry. Percentages of $S$ phase (A) and actively DNA-replicating (B) lung cells generated from p53-null and p53R172H-KI mice and their frequency of mitotic entry (C) at 12 and 16 hours after density arrest and replating are shown by bar graphs. S phase and DNA-replicating cells were identified by PI staining or BrdU pulse labeling and PI staining followed by flow cytometry. Gating is shown in Supplemental Figure 4. Mitotic cells were identified by immunostaining microtubules with FITC-labeled $\alpha$-tubulin antibody and staining DNA with DAPI followed by confocal microscopy (images shown in Supplemental Figure 5). Approximately 500 cells were counted in each sample. The $P$ value calculated using Student's $t$ test is shown at the bottom of the bar graph. Each experiment was repeated 3 times, and representative data are shown.

red and green fluorescence-tagged antibodies, respectively, followed by confocal microscopy. Scoring of bidirectional origins in untangled immunostained DNA fibers (Supplemental Figure 2, A and $B$ ) revealed that the frequency of origin firing in lung cells from p53R172H-KI mice was at least double of that in lung cells from p53-null mice (Figure 1, A-C), whereas frequency of origin firing in replicating lung cells isolated from normal mice (WT p53) was approximately 3 -fold lower than that in p53-null mice (Figure 1B) as expected. These results suggest that the presence of the GOF p53 mutant $\mathrm{R} 172 \mathrm{H}$ in nontransformed lung cells accelerates the frequency of origin firing at early $\mathrm{S}$ phase.

Knockdown of GOF $p 53$ mutant in human lung cancer cells reduces the frequency of origin firing. The ability of GOF p53 mutants to increase origin firing in a human lung cancer cell line that naturally expresses GOF p53 was also determined. For this purpose, endogenous p53R273C expression in the human SCLC lung cancer cell line $\mathrm{H} 1048$ or R175H expression in the human non-small cell lung cancer (NSCLC) VMRC-LCD cell line (http://p53.iarc.fr/ CellLines.aspx) was stably knocked down by expressing shRNA against p53 (shp53) or a control shRNA against GFP (shGFP) from a lentiviral expression vector (16). H1048 and VMRC cells stably expressing shGFP or shp53 were synchronized by density arrest and replating. Fiber analysis of replicating DNA from these cells was performed as described above. Scoring of bidirectional origins in untangled immunostained DNA fibers showed that knockdown of endogenous p53 significantly reduced frequency of origin firing in both the cell lines (Figure 1, D-G). These results indicate that GOF p53 mutants accelerate firing of DNA replication origins at early $S$ phase in cells harboring p53 mutation.

GOF 553 upregulates expression of intra-S phase checkpoint kinase CHK1, preventing collapse of replication forks. Hyperreplicative activities of oncogenes induce cellular checkpoint responses $(31,32)$ to prevent collapse of progressing replication forks and thus cell death $(22,24,33,34)$. Intra-S phase CHK1 is known to protect replication forks, while inactivation of CHK1 leads to fork collapse, which could be evidenced by phosphorylation of $\gamma \mathrm{H} 2 \mathrm{AX}(22,24,33)$.
Since GOF p53 accelerates origin firing at early S phase, its ability to activate intra-S phase checkpoint for preventing collapse of replication forks in cells harboring the mutation was investigated. For this purpose, lung cells from p53-null and p53R172H-KI mice were partially synchronized by density arrest and replating and the cell extracts were analyzed for CHK1 expression and phosphorylation at $\mathrm{S} 345$ by immunoblot analysis. Results (Figure 2A) of these experiments revealed that in comparison with lung cells from $p 53^{-/-}$mice, lung cells from p53R172H-KI mice showed higher levels of phosphorylated CHK1 (p-CHK1) (9- to 10-fold) and CHK1 (2- to 3-fold) at early $S$ phase in repeated experiments, suggesting that GOF p53 may increase CHK1 phosphorylation during increased origin firing by upregulating CHK1 expression.

Since GOF 553 increases CHK1 and p-CHK1 levels, the oncoprotein may reduce replication fork collapse and therefore $\gamma \mathrm{H} 2 \mathrm{AX}$ foci formation. The presence of chromatin-associated $\gamma \mathrm{H} 2 \mathrm{AX}$ in lung cells from p53-null and p53R172H-KI mice was determined by acid extraction of $\gamma \mathrm{H} 2 \mathrm{AX}$ from the chromatin fractions and immunoblot analysis. The result of this experiment showed that, compared with lung cells from p53-null mice, chromatin-associated $\gamma \mathrm{H} 2 \mathrm{AX}$ levels in lung cells from p53R172H-KI mice were drastically reduced (Figure 2, A and B). Similar analysis using mock-depleted or p53-depleted H1048 human lung cancer cells stably expressing nontargeting shGFP or shp53 showed reductions in p-CHK1 and CHK1 levels and increases (approximately 2- to 6-fold) in $\gamma \mathrm{H} 2 \mathrm{AX}$ levels in shp53-expressing $\mathrm{H} 1048$ cells compared with $\mathrm{H} 1048$ cells expressing shGFP (Figure 2, C and D). These results indicate that expression of GOF p53 increases p-CHK1 and CHK1 levels and reduces chromatin-bound $\gamma \mathrm{H} 2 \mathrm{AX}$ levels, signifying that checkpoint activation by GOF p53 prevents collapse of replication forks and thus $\gamma \mathrm{H} 2 \mathrm{AX}$ foci formation.

Next, the ability of GOF p53 to reduce frequency of replication fork collapse was tested. For this purpose, partially synchronized lung cells from p53-null and p53R172H-KI mice were sequentially labeled for 20 minutes with IdU and 20 minutes with CldU at early $\mathrm{S}$ phase. Replicating fibers and direction of fork progression were 
A

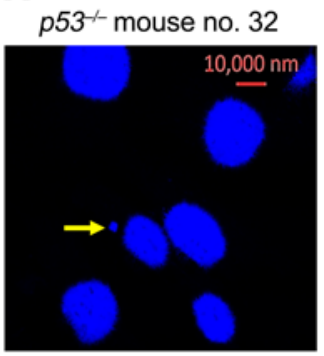

C

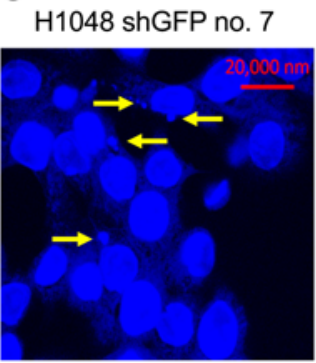

$\mathrm{R} 172 \mathrm{H}$ mouse no. 55

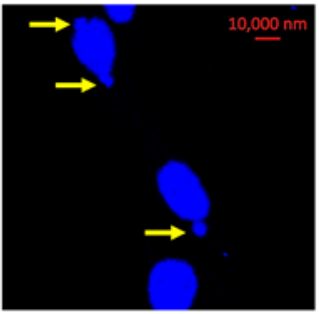

H1048 shGFP no. 1

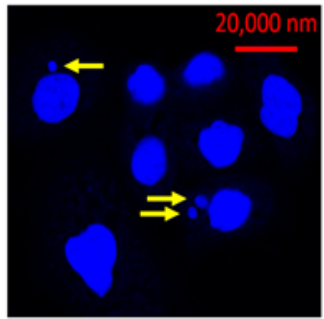

p53-- mouse no. 34

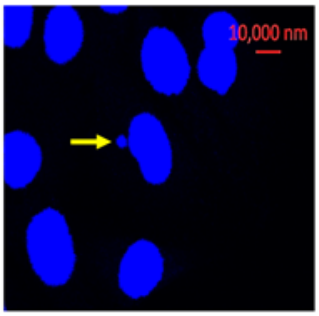

H1048 shp53 no. 19

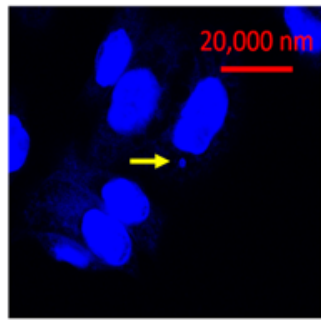

R172H mouse no. 67

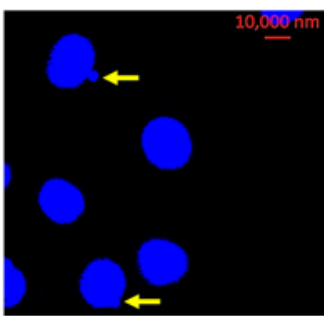

H1048 shp53 no. 1

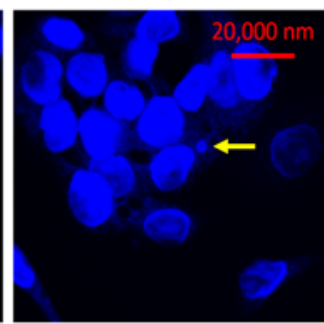

B

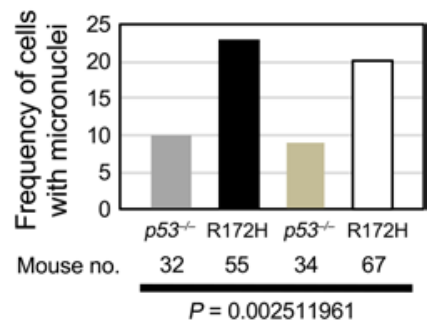

D

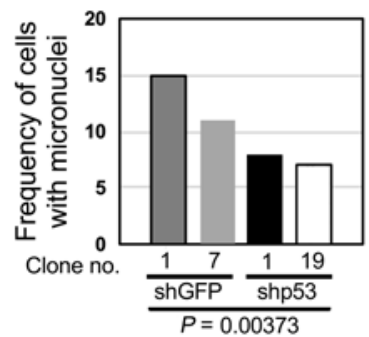

Figure 4. Lung cells with GOF p53 form micronuclei at a higher frequency than lung cells from p53-null mice. Representative images of micronuclei (indicated by arrows) from lung cells of $p 53^{-/-}$and p53 R 172H-KI (R172H) mice (A) and mock-depleted (shGFP) or p53-depleted (shp53) H1048 lung cancer cells (C), detected by confocal microscopy after DAPI staining. Original magnification, $\times 40$. Bar graphs $(\mathbf{B}$ and $\mathbf{D})$ compare the percentages of cells with micronuclei in each sample. Lung cells from 2 mice of each construct or 2 clones stably expressing shGFP or shp53 were analyzed. Five hundred cells were scored for each sample. $P$ values calculated using the $\chi^{2}$ test are shown at the bottom of the bar graphs. Each experiment was repeated twice, and representative data are shown.

detected in these cells by immunostaining IdU and CldU with red and green fluorescence-tagged antibodies, respectively. It was reasoned that if the progressing forks collapse during the incorporation of IdU, these forks should not incorporate CldU and, therefore, the red-only tracks should indicate collapse of progressing replication forks (Figure 2E). Untangled replicating DNA fibers were scored for the presence of red-only tracks. Results of this experiment revealed that, in comparison with lung cells from $\mathrm{p} 53$ null mice, the frequency of red-only tracks was drastically reduced in lung cells from p53R172H-KI mice (Figure 2, E and F), indicating a reduction in fork collapse in lung cells expressing p53R172H.

Reduction of replication fork collapse due to activation of CHK1 expression by GOF p53 should reduce chromatin association of $\gamma \mathrm{H} 2 \mathrm{AX}$. To complete the analysis of GOF p53-mediated modulation of fork collapse, this possibility was examined. For this experiment, cultured lung cells from p53R172H-KI mice were treated with a CHK1 inhibitor, PF00477736. Chromatinassociated $\gamma \mathrm{H} 2 \mathrm{AX}$ was extracted and analyzed by immunoblot analysis. Results of this experiment indicated that the CHK1 inhibitor PF00477736 elevated the levels of chromatin-associated $\gamma \mathrm{H} 2 \mathrm{AX}$ more than 6-fold (Figure 2, $\mathrm{G}$ and $\mathrm{H}$ ) as opposed to a 1.6fold increase in p53-null lung cells. Consistent with earlier studies $(35,36)$, inhibition of CHK1 upregulated RPA phosphorylation (at Ser4 and Ser8) due to generation of replication stress (Figure $2 \mathrm{G})$. These data indicate that GOF p53-mediated reduction in $\gamma \mathrm{H} 2 \mathrm{AX}$ can be overridden by inhibition of CHK1. These observations signify that cell proliferation induced by GOF $\mathrm{p} 53$ is at least partly due to its ability to increase expression of CHK1, the activity of which prevents collapse of an increased number of replication forks generated by the mutant protein, allowing rapid completion of genome duplication and cell proliferation sooner than p53-null cells. Thus, the ability of GOF p53 to upregulate CHK1 expression, and therefore its activity, may contribute to its ability to confer an addictive influence to the cells expressing the mutant protein.

GOF $p 53$ promotes rapid genome duplication and mitotic entry. Increased number of origin firing and successful progression of generated replication forks should lead to rapid genome duplication. Therefore, we determined whether GOF p53 promotes quicker genome duplication in comparison with p53-null cells by analyzing $\mathrm{S}$ phase progression and mitotic entry of partially synchronized lung cells from p53-null and p53R172H-KI mice generated as described above. Flow cytometric analysis of propidium iodide-stained (PI-stained) cells at 12 and 16 hours after release from density arrest showed that lung cells from both p53R172H-KI and p53-null mice entered $\mathrm{S}$ phase at a similar time and frequency, with approximately $29 \%$ of p53R $172 \mathrm{H}-\mathrm{KI}$ and $21 \%$ of p 53 -null cells in the $\mathrm{S}$ phase at 12 hours after release. However, with progress

Table 1. An inhibitor of origin firing reduces micronuclei formation in lung cells of p53R172H-KI mice

$\begin{array}{ccc}\text { Mouse number } & \text { Treatment } & \begin{array}{c}\text { Nuclei with micronuclei } \\ \text { (500 scored) }\end{array} \\ 55 & \text { DMSO } & 36 \\ & \text { PHA76749 }(6 \mu \mathrm{M}) & 25 \\ 67 & \text { DMSO } & 39 \\ & \text { PHA76749 }(6 \mu \mathrm{M}) & 27\end{array}$

$P=0.0014249$. 
A $22 / 23$

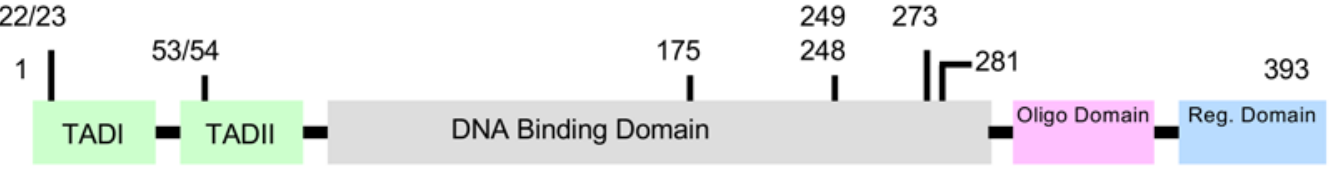

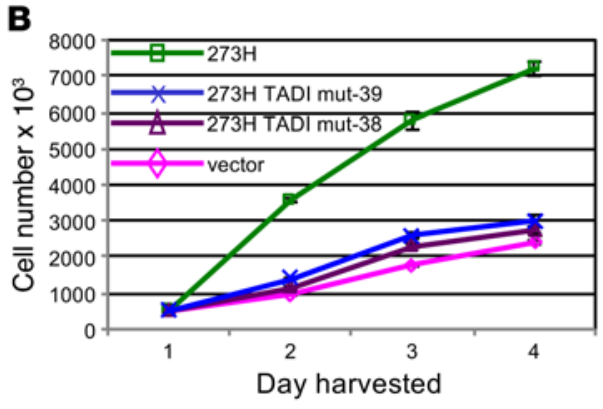

C

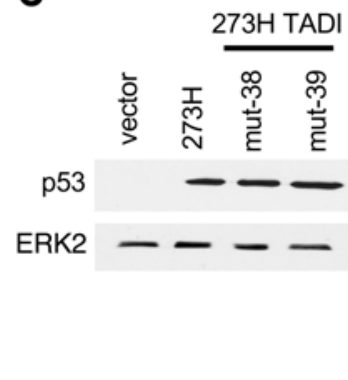

D

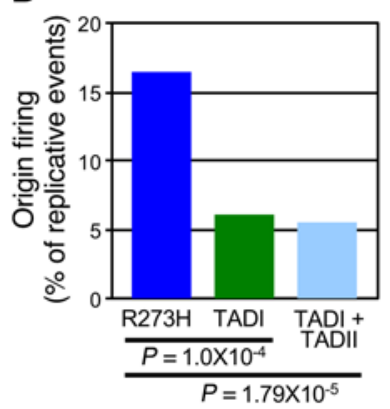

$\mathbf{E}$

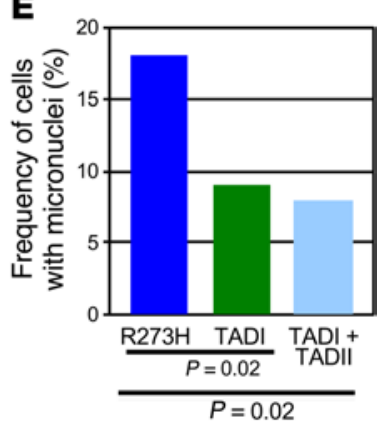

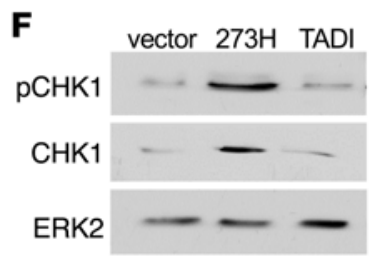
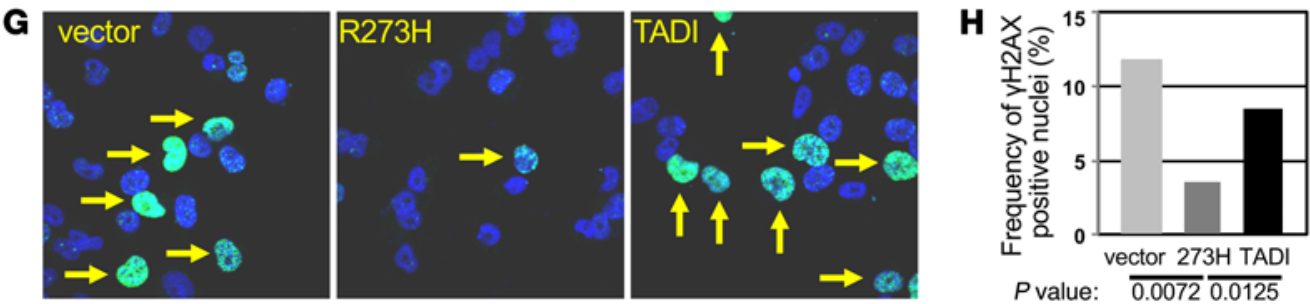

Figure 5. The transactivation function of GOF p53 is required for cell proliferation, increased frequency of replication origin firing, micronuclei formation, CHK1 expression and phosphorylation, and replication fork protection. (A) Schematic showing functional domains of p53, including its TADI and TADII, sites of hot spot mutations, and created mutations on TADI (L22Q and W23S) and TADII (L53Q and F54S). Reg, regulatory. (B) Rate of proliferation of $\mathrm{H} 1299$ cells stably expressing $\mathrm{p} 53 \mathrm{R} 273 \mathrm{H}$ or its TADI mutant was determined. Data are presented as mean \pm SD of triplicate experiments. Two clones of TADI mutants were used. (C) p53 expression was confirmed by immunoblot analysis. (D) Origin firing was determined by fiber analysis of replicating DNA. Data obtained by scoring 200 untangled fibers are shown by a bar graph. $P$ value calculated using Student's $t$ test is shown at the bottom of the graph. (E) Micronuclei formation was determined by DAPI staining. Data obtained by scoring 500 cells are shown by bar graphs. $P$ value calculated using $\chi^{2}$ test is shown at the bottom of the bar graphs. (F) Expression of CHK1 and p-CHK1 (at S345) in H1299 cells expressing p53R273H or its TADI mutant was determined by immunoblot analysis. (C) $\gamma \mathrm{H} 2 \mathrm{AX}$ foci formation in $\mathrm{H} 1299$ cells expressing p53R273H or TADI mutant was determined by immunostaining with Alexa Fluor 488-coupled $\gamma \mathrm{H} 2 \mathrm{AX}$ antibody followed by confocal microscopy. Arrows indicate nuclei with green foci. Original magnification: $\times 20$. (H) Frequency of nuclei with $\gamma \mathrm{H} 2 \mathrm{AX}$ foci in each construct is shown by bar graph; 250 cells were scored, and $P$ value was calculated using Student's $t$ test. Each experiment was repeated 3 times.

through $\mathrm{S}$ phase, at 16 hours, lung cells from p53-null mice accumulated in S phase $(43.5 \%)$, whereas lung cells from p53R172H$\mathrm{KI}$ mice progressed to $\mathrm{G}_{2} / \mathrm{M}$, showing $29.6 \% \mathrm{~S}$ phase cells (Figure $3 \mathrm{~A})$. Accordingly, at 16 hours, $23.7 \%$ of p53R172H-KI cells completed genome duplication and entered $\mathrm{G}_{2} / \mathrm{M}$ phase as opposed to $15.3 \%$ of lung cells from p53-null mice (Supplemental Figure $4, \mathrm{~A}-\mathrm{D})$. These data were further confirmed by identifying replicating cells at 12 and 16 hours after release from density arrest by pulse labeling with BrdU followed by PI staining. Flow cytometric analysis (Supplemental Figure 4, E and F) showed that, consistent with the data shown in Figure 3A, similar percentages of lung cells from p53R172H-KI or p53-null mice incorporated BrdU (18.8\% and $16 \%$, respectively) at 12 hours. However, at 16 hours, $28.5 \%$ of p53-null cells incorporated BrdU, most (90\%) of which remained in early or mid-S phase. In contrast, only $10.8 \%$ of lung cells from p53R172H-KI mice were found in early and mid-S phase (Figure 3B). Next, mitotic entry of the lung cells at 16 hours after release of density-arrested cells was determined. Mitotic cells (Supplemental Figure 5) were identified by immunostaining microtubules with $\alpha$-tubulin antibody and staining nuclei with DAPI. Percentages of cells in mitosis were determined by scoring 500 cells for mitotic nuclei under a fluorescent microscope. The results revealed that lung cells from p53R172H-KI mice entered mitosis at a 2.5to 2.8-fold higher frequency than did p53-null lung cells (Figure 3C). These results show that p53-null lung cells take longer times to complete genome duplication to enter mitosis compared with p53R172H-KI lung cells, which complete genome duplication and enter mitosis sooner than p53-null lung cells.

GOF $p 53$ induces micronuclei formation, which can be prevented by blocking origin firing. Increased frequency of origin firing has been implicated in genomic abnormality $(22,24,37-40)$, and one of the indicators of genomic abnormality is micronuclei formation (41-43). Although an increase in CHK1 levels would protect collapse of replication forks stalled due to increased origin firing, it has been reported that the topological stress associated with progress of an increased number of replication forks may lead to genome abnormality by mechanisms such as fork reversal, which could be evidenced by micronuclei formation $(39,40)$. Since GOF 
A

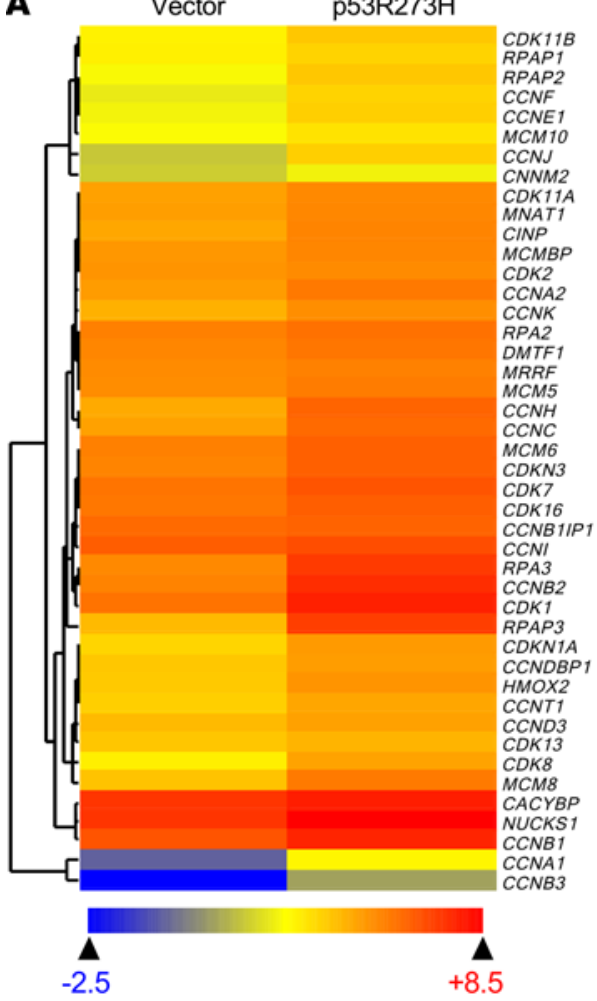

B

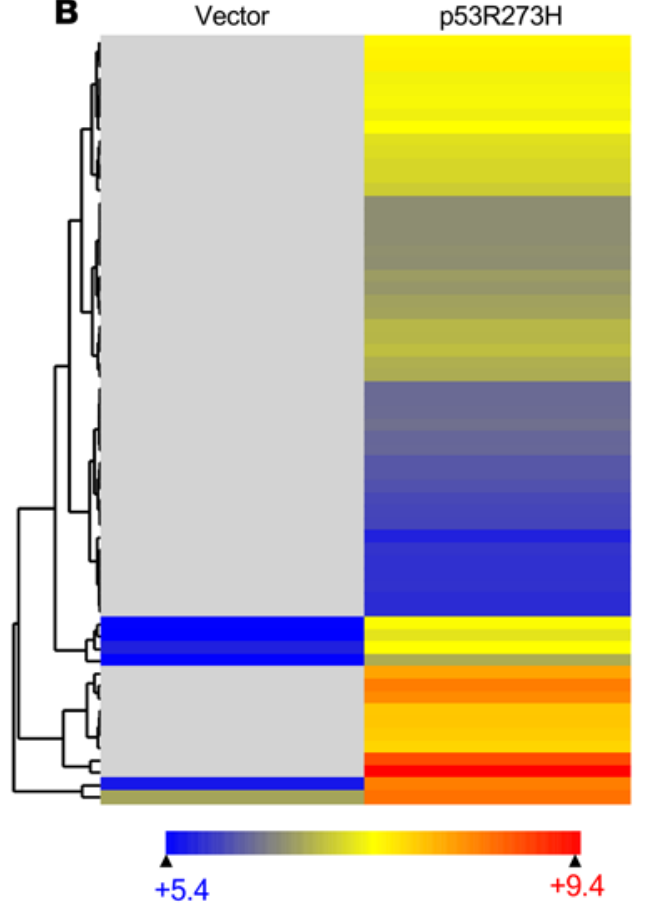

C

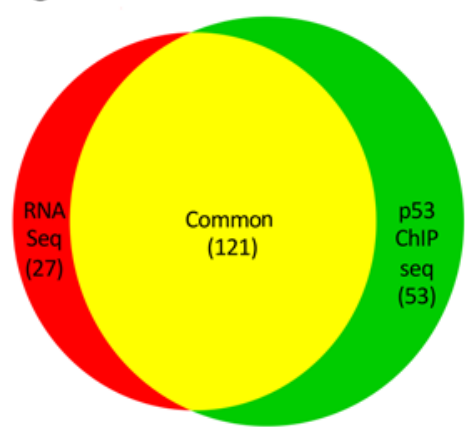

Figure 6. GOF p53 upregulates expression of an array of genes involved in regulation of cell cycle and DNA replication and localizes on their upstream regulatory sequences. Heat maps of transcripts generated from RNA-Seq analysis (A) and DNA fragments generated from ChIP-Seq analysis (B) of H1299 cells stably transfected with vector (Vector) and stably expressing p53R273H (R273H). Number of sequences identified in B was too numerous to be listed in the figure. Gray areas indicate no $\mathrm{p} 53$ binding. A Venn diagram (C) shows 121 genes common between the RNA-Seq and ChIP-Seq gene list, representing $81.75 \%$ of the RNA-Seq genes and $69.54 \%$ of the p53 ChIP-Seq genes that are involved in cell cycle and DNA replication. Genes that showed greater than 2-fold upregulation by expression of the p53R273H mutant and whose $P$ value was less than 0.01 were considered significant.

p53 increases origin firing, the frequency of micronuclei formation in the presence or absence of GOF p53 was determined. For this purpose, cultured lung cells generated from p53-null or p53R172HKI mice were plated on coverslips, fixed and stained with DAPI, and cells with micronuclei were scored by confocal microscopy. Scoring of cell nuclei associated with micronuclei revealed that lung cells from $\mathrm{p} 53 \mathrm{R} 172 \mathrm{H}-\mathrm{KI}$ mice formed micronuclei at a significantly higher frequency than lung cells from p53-null mice (Figure 4, A and B). Furthermore, it was determined whether knockdown of p53 reduces micronuclei formation in human lung cancer cells. H1048 cells (p53R273C) expressing shGFP or shp53 were cultured on coverslips, fixed and stained with DAPI, and scored for cells with micronuclei. Results of this experiment revealed a significant reduction in the frequency of cells generating micronuclei in H1048 cells stably expressing shp53 compared with those expressing shGFP (Figure 4, C and D). These data indicate that GOF p53 increases micronuclei formation, an index of genetic abnormality.

Next, experiments were designed to determine whether increases in the frequency of origin firing elevate micronuclei formation by GOF p53. It is known that in mammalian cells, DNA replication origins are activated by cyclin-dependent kinases (CDK) and CDC7 kinase, which induces melting of double-stranded DNA at the origin of replication $(44,45)$. Since GOF 553 induces origin firing, a CDC7 kinase inhibitor, PHA767491, which specifically prevents activation of origins but does not prevent fork progression $(46,47)$, was used to determine whether inhibition of origin firing in lung cells harboring a GOF p53 mutation would reduce frequency of micronuclei formation. Lung cells from p53R172H-KI mice were treated with PHA76749 or vehicle (DMSO) for 3 replication cycles (approximately 72 hours), and the frequency of micronuclei formation was determined as described above. The results (Table 1) of this experiment showed reduction in percentages of nuclei associated with micronuclei in lung cells from p53R172H-KI mice. These data indicate that inhibition of origin firing in lung cells from p53R172H-KI mice reduces their ability to generate micronuclei.

The transactivation function of GOF $p 53$ mutants is required for GOF p53's ability to accelerate cell growth, increase firing of DNA replication origins, form micronuclei, and protect replication forks. GOF p53 mutants activate transcription of growth-promoting genes using GOF p53's transactivation domains (TADs) (Figure $5 \mathrm{~A}$; refs. $12,13,48,49)$. The transactivation function of GOF p53 mutants has been related to the ability of GOF p53 to induce tumorigenesis and cell proliferation $(50,51)$. Therefore, experiments were designed to investigate whether the transactivation function of GOF p53 is also required for its ability to increase cell growth, origin firing, micronuclei formation, and CHK1 expression and to reduce $\gamma \mathrm{H} 2 \mathrm{AX}$ foci formation. For this purpose, plasmids expressing $\mathrm{p} 53 \mathrm{R} 273 \mathrm{H}$ or $\mathrm{p} 53 \mathrm{R} 273 \mathrm{H}$ with compromising mutations (Fig- 
A

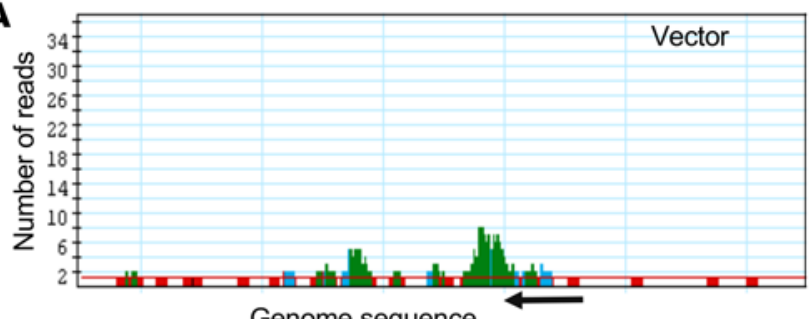

Genome sequence

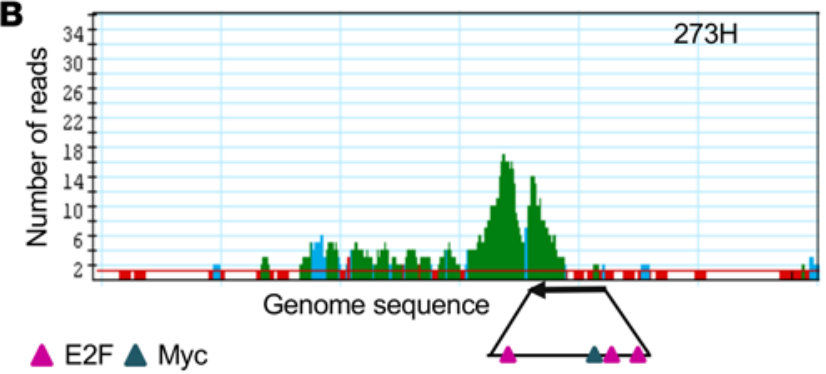

D

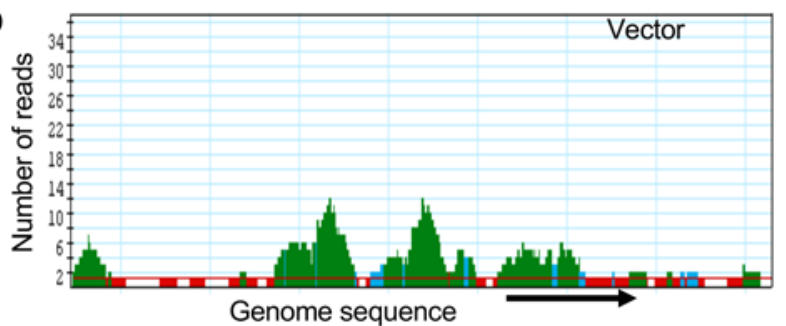

E

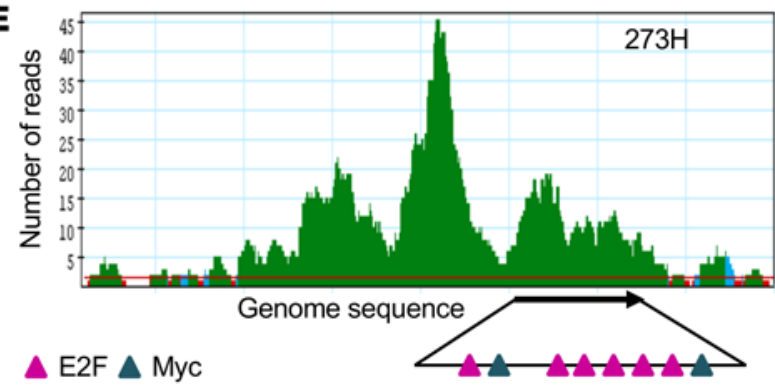

C

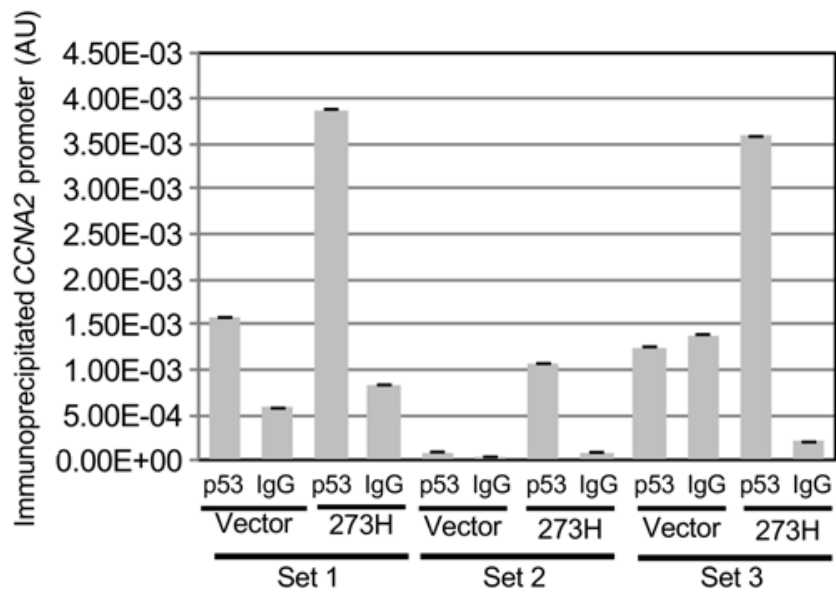

$\mathbf{F}$

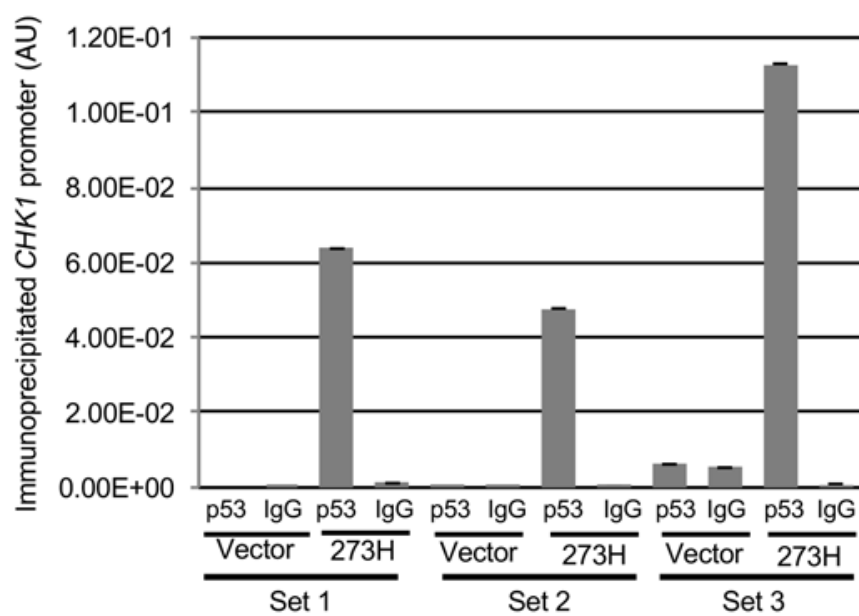

Figure 7. GOF p53 localizes on the upstream sequences of CCNA2 and CHK1 genes. The peaks represent areas under which maximal p53R273H binding occurs, as apparent by next-generation sequence analysis of ChIP fragments. Peaks of ChIP fragments on CCNA2 promoter from H1299 cells stably transfected with control vector (A) or stably expressing p53R273H (B). ChIP analysis (C) to confirm localization of p53R273H on CCNA2 promoter. Peaks of ChIP fragments on CHK1 promoter from H1299 cells stably transfected with control vector (D) or stably expressing p53R273H (E). ChIP analysis (F) to confirm localization of p53R273H on CHK1 promoter. Results from triplicate sets of extracts of H1299 cells expressing p53R273H (273H) or empty vector are shown by bar graphs as mean \pm SEM. ChIP using IgG control (IgG) has been included in each case. The region of genome showing p53R273H-specific binding as determined by ChIP and the direction of coding sequences are indicated by arrows. Blue and purple arrowheads indicate Myc- and E2F-binding sites, respectively, on the identified promoter sequences.

ure 5A) of TADI (L22Q, W23S) or TADI and TADII (L22Q, W23S, L53Q, F54S) were stably introduced into p53-null H1299 human lung cancer cells, and the ability of the stable transfectants to perform the above biological activities was determined. Examination of the rate of cell proliferation revealed that H1299 cells expressing p53R273H proliferated more rapidly than cells expressing either empty vectors or TADI mutants of p53R273H (Figure 5, B and C). Fiber analysis of replicating DNA from H1299 cells expressing p53R273H, p53R273HTADI, or p53R273HTADI/TADII at early S phases showed that $\mathrm{H} 1299$ cells expressing p53R273H fired almost 3-fold more origins than either of the TAD mutants (Fig- ure 5D). This observation indicates that to induce origin firing, p53R273H requires its transactivation function.

The ability of the transactivation-deficient p53R273HTADI or TADI/TADII mutants to induce micronuclei formation was determined by staining of fixed cells on coverslips with DAPI and scoring cells with micronuclei by confocal microscopy. The results revealed that H1299 cells expressing transactivation-deficient p53R273HTADI or TADI/TADII formed micronuclei less frequently than H1299 cells expressing transactivation-competent p53R273H (Figure 5E). Consistently, transactivation-deficient p53R273HTADI did not activate expression and phosphorylation 
A

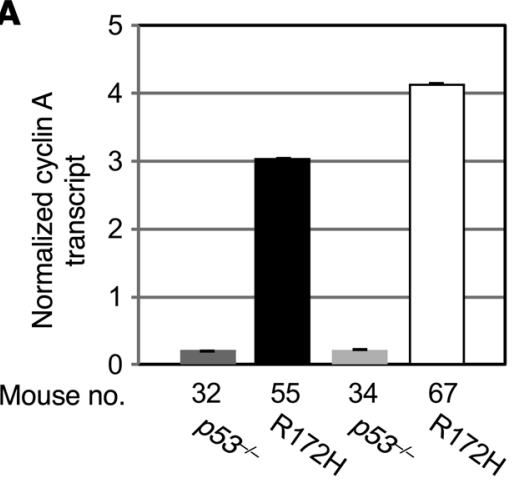

D

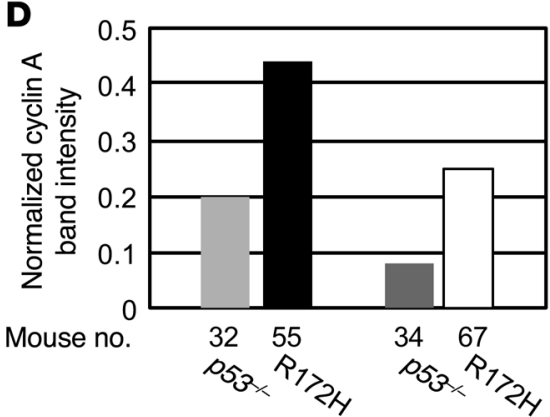

B

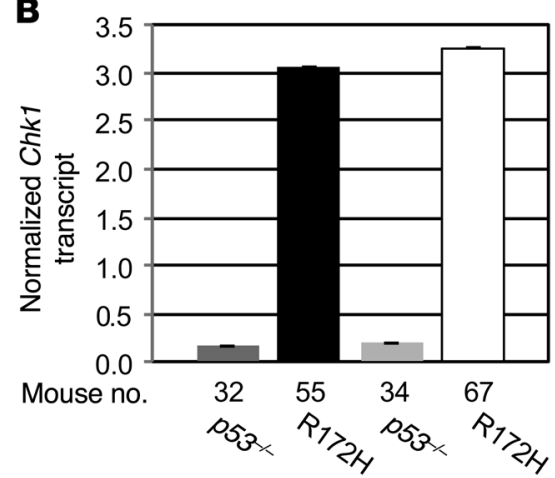

C

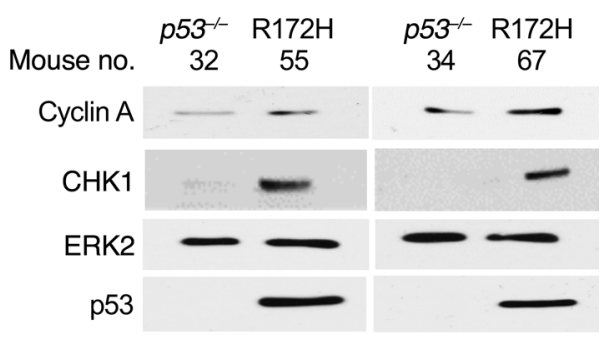

$\mathbf{E}$

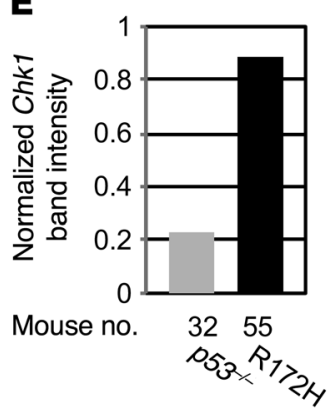

$\mathbf{F}$

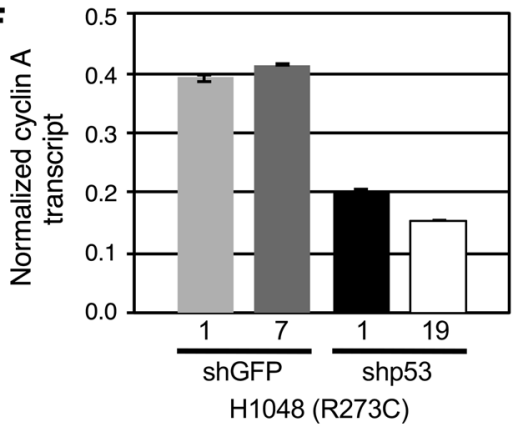

G

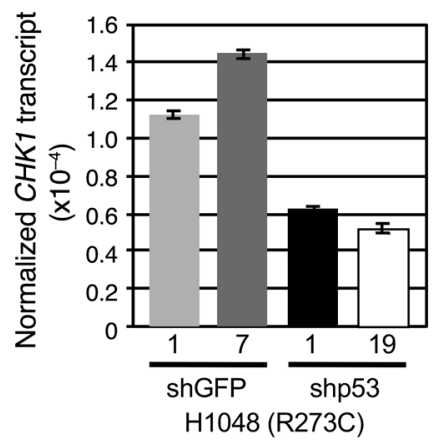

H

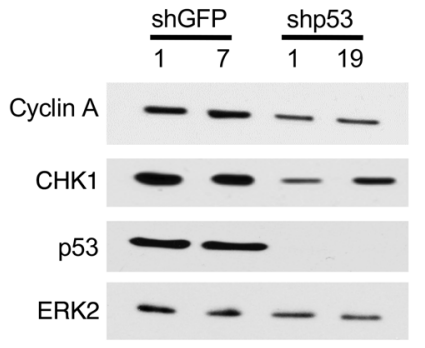

I

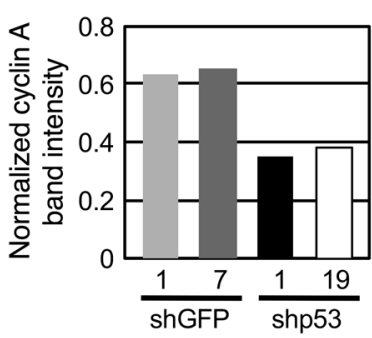

J

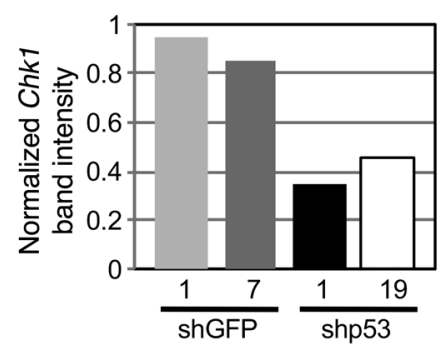

Figure 8. GOF p53 induces cyclin A and CHK1 expression. Transcript levels of cyclin A and CHK1 in lung cells from p53-null or R172H-KI (R172H) mice (A and B) and H1048 human lung cancer cells stably expressing shRNA against GFP or p53 (F and G), determined by RT-QPCR. Data are plotted as mean \pm SEM. Cyclin A and CHK1 protein levels (C-E and $\mathbf{H}-\mathbf{J})$ were determined by immunoblot analysis. Bar graphs show band intensities of cyclin A ( $\mathbf{D}$ and $\mathbf{I})$ and $\mathbf{C H K 1}$ (E and J) determined by densitometry and normalized by ERK2 loading control. Lung cells from 2 mice of each construct or 2 clones stably expressing shGFP or shp53 were analyzed. Each experiment was repeated 3 times.

of CHK1 (Figure 5F). Immunostaining of $\gamma \mathrm{H} 2 \mathrm{AX}$ foci showed a drastic increase in foci formation in H1299 cells expressing transactivation-deficient p53R273H TADI mutants compared with H1299 cells expressing p53R273H (Figure 5, G and H). These results indicate that the ability of GOF p53 mutants to accelerate cell proliferation, increase origin firing and micronuclei formation, upregulate expression and phosphorylation of CHK1, and protect fork collapse requires its transactivation function.

GOF 553 mutants upregulate expression of genes involved in cell cycle regulation and DNA replication. Several laboratories have demonstrated that GOF p53 accelerates cell proliferation and tumor growth in comparison with p53-null or GOF p53-knockdown cells (12, 15, 16). Since the data presented above indicate that GOF $\mathrm{p} 53$ requires its TADs to increase origin firing and to prevent replication fork col- lapse, the profile of genes activated by GOF p53 mutant R273H was determined by RNA-Seq analysis. RNA was extracted from H1299 cells stably expressing p53R273H or stably transfected with empty vector, and the expression profiles were compared. To determine the mechanism by which GOF p53 mutants increase origin firing and prevent collapse of replication forks, profiles of genes involved in the regulation of DNA replication were analyzed. The analysis revealed that, indeed, GOF p53 mutants upregulated an array of genes that regulate cell cycle and DNA replication (Figure 6A).

GOF 553 mutants recognize regulatory elements upstream of cell cycle and DNA replication genes. To determine whether GOF 553 mutants localize on the regulatory elements of the DNA replication genes, ChIP using H1299 cells stably expressing p53R273H was performed. Chromatin fragments bound to p53R273H were 

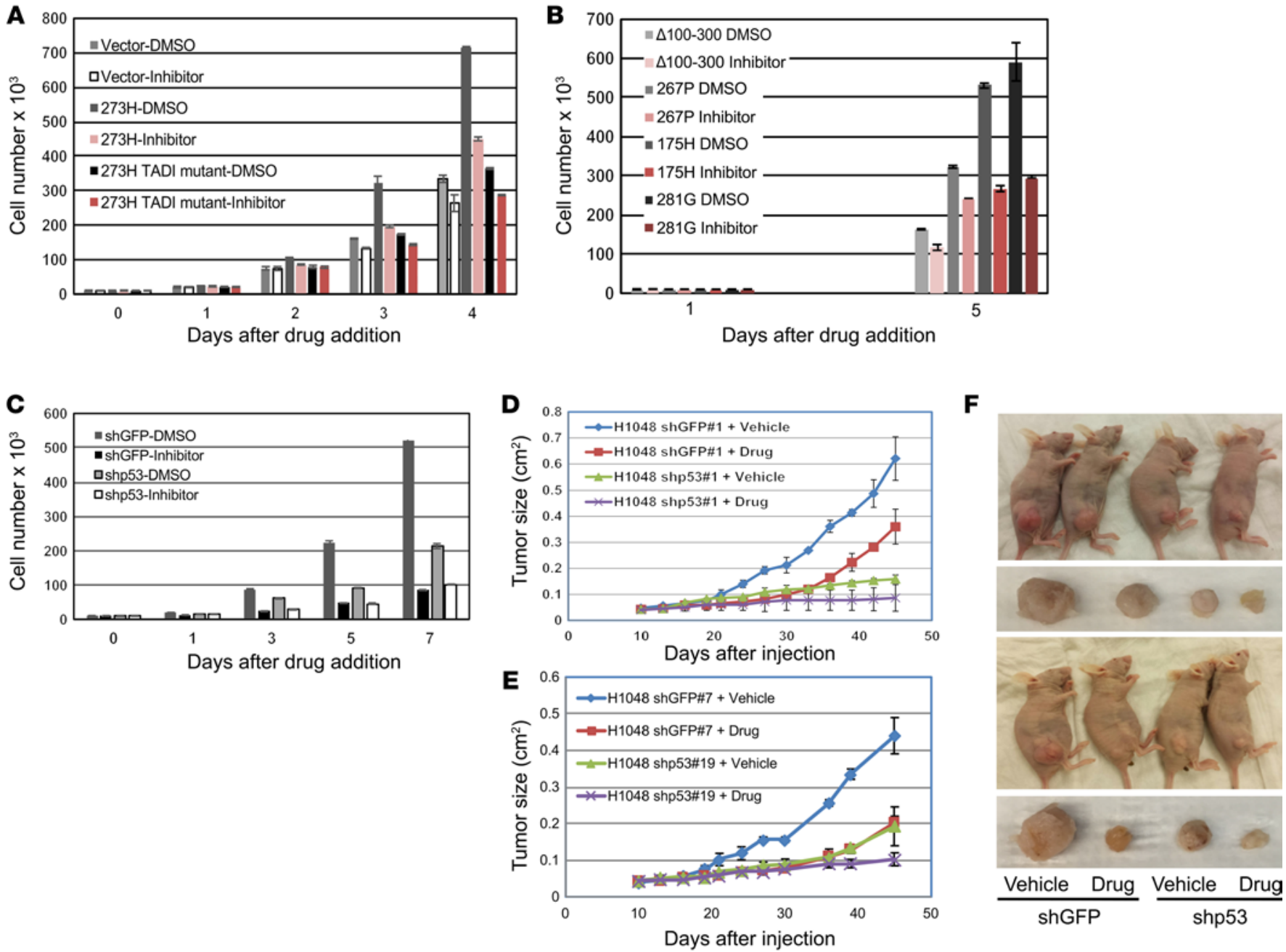

Figure 9. A small molecule inhibitor of CHK1 selectively inhibits growth and reduces tumor size of lung cancer cells with p53 mutation. Rate of proliferation of (A) H1299 cells stably expressing p53R273H or its TADI mutant or a vector control; (B) proliferation of H1299 cells stably expressing p53 mutants $281 \mathrm{C} \Delta 100-$ 300, 267P, 175H, and 281G; and (C) H1048 lung cancer cells stably expressing shp53 or shGFP in the presence of a CHK1 inhibitor PF00477736 (100 nM) (Inhibitor) or only solvent (DMSO). Data are shown as mean \pm SD from triplicate experiments. Rate of tumor growth (D and E) from H1048 cells expressing shGFP or shp53 treated with vehicle or PF00477736, $10 \mathrm{mg}$ per kg (drug). Data from 2 sets of clones (D and E) and representative photographs (F) of tumors are shown. Representative data from $n=4$ mice in each experimental group are plotted as mean \pm SEM. Assay was repeated in $n=12$ mice per experimental group.

pulled down using 2 p53 antibodies, DO1 and FL393. Pulled down fragments were analyzed by ChIP-Seq. H1299 cells stably transfected with vector and IgG isotypes of the antibodies were used as controls. Four to ten million reads obtained from Illumina HiSeq were analyzed using the ArrayStar 11 (DNASTAR) program. The analysis revealed that $\mathrm{p} 53 \mathrm{R} 273 \mathrm{H}$ localizes on the upstream regulatory sequences of an array of genes involved in cell cycle regulation and DNA replication (Figure 6B). A Venn diagram (Figure 6C) shows that there are 121 genes in common between RNA-Seq and p53 ChIP-Seq data, representing $81.75 \%$ of the genes detected by RNA-Seq and $69.54 \%$ of the genes detected by 53 ChIP-Seq that are involved in cell cycle and DNA replication, 2 of which are cyclin A and CHK1. Localization of $\mathrm{p} 53 \mathrm{R} 273 \mathrm{H}$ on the regulatory regions of some of the replication genes identified by ChIP-Seq and their activated expression detected by RNA-Seq were confirmed by ChIP and quantitative reverse-transcriptase PCR (RT-QPCR), respectively (Supple- mental Figure 6). These results strongly support the hypothesis that $\mathrm{p} 53 \mathrm{R} 273 \mathrm{H}$ activates expression of DNA replication genes by targeting their regulatory sequences.

GOF p53 mutants recognize upstream regulatory sequences of CCNA2 and CHK1 transcription start sites. The ChIP-Seq analysis described above detected localization of p53R273H on the upstream sequences of the CCNA2 and CHK1 genes, expression of which are needed for firing of DNA replication origins (19-21) and stability of initiated replication forks (22-24), respectively. Therefore, experiments were performed to confirm localization of p53R273H on the upstream regulatory sequences of these genes. ChIP analysis (Figure 7) showed that an anti-p53 antibody immunoprecipitated DNA fragments spanning a 335-bp region 106 bp upstream of the CCNA2 transcription start site (Figure 7, A-C) and a 1000-bp region $200 \mathrm{bp}$ upstream of the $C H K 1$ transcription start site (Figure 7, D-F). These data show that p53R273H localizes on the upstream sequences of the CCNA2 and CHK1 transcription start sites. 
A Cells with GOF p53

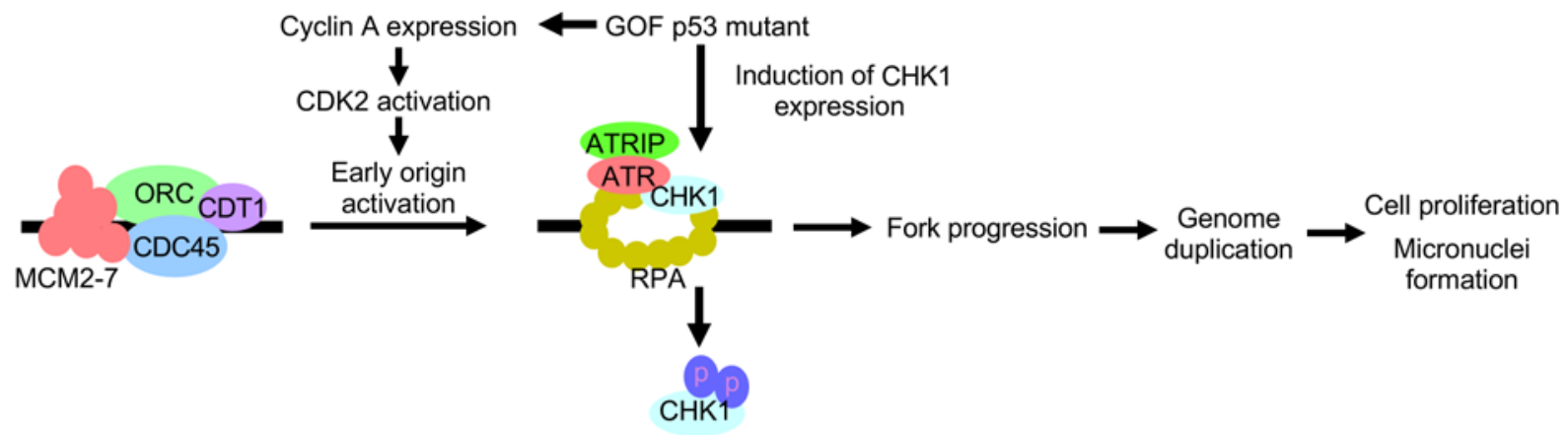

B p53-null cells

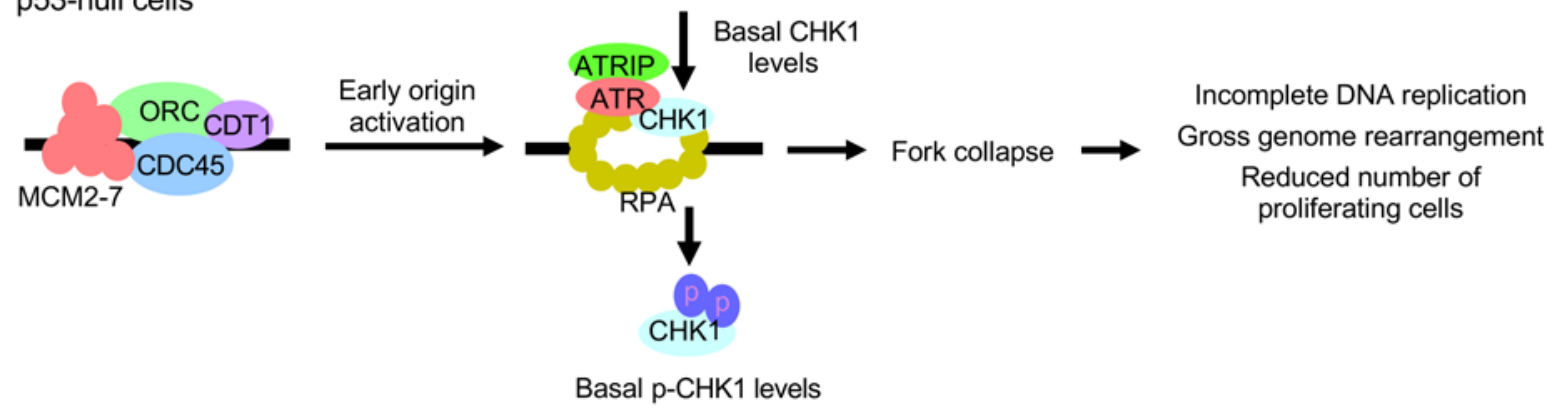

Figure 10. GOF p53 promotes inaccurate genome duplication, whereas p53-null cells show collapse of replication forks. The downstream consequences of unscheduled origin firing in (A) GOF p53-expressing and (B) p53-null lung cells are depicted.

GOF 553 mutants activate expression of cyclin A and CHK1 at early $S$ phase. Since RNA-Seq data (Figure 6A) presented above suggest that GOF p53 increases cyclin A and CHK1 expression and GOF p53 recognizes their upstream regulatory sequences (Figure 7), experiments were performed to confirm whether GOF p53 upregulates cyclin A and CHK1 expression at early S phase in the absence of other known gene mutations. Cell cycle-dependent expression of cyclin A and CHK1 in partially synchronized lung cells from p53-null and p53R172H-KI mice at early S phase were compared. RT-QPCR of transcripts and immunoblot analysis of extracts prepared from these cells showed a significant increase in cyclin A and CHK1 expression at the RNA (Figure 8, A and B, respectively) and protein levels (Figure 8, C-E) in lung cells from p53R172H-KI mice in comparison with lung cells from p53-null mice. These data are consistent with the immunoblot analysis (Figure 2A), which showed that lung cells from p53R172H-KI mice express higher levels of CHK1 in comparison with lung cells from $\mathrm{p53}$-null mice, suggesting that an increase in CHK1 expression leads to an increase in p-CHK1.

Knockdown of GOF $p 53$ diminishes cyclin $A$ and $C H K 1$ expression at early $S$ phase in a human lung cancer cell line. To further confirm whether GOF p53 increases cyclin A and CHK1 expression at early S phase, GOF p53 was knocked down in H1048 lung cancer cell lines. H1048 cells stably expressing control shGFP or shp53 RNA were partially synchronized by confluence arrest and replating. Expression of cyclin A and CHK1 was analyzed at early S phase. RT-QPCR of transcripts and immunoblot analysis of cell extracts prepared from these cells showed that knockdown of GOF p53 significantly reduced cyclin A and CHK1 expression at the RNA (Figure 8, F and $\mathrm{G}$ ) and protein levels (Figure 8, $\mathrm{H}-\mathrm{J}$ ). Consistently, in a similar experiment, knockdown of p53R175H in VMRC human lung cancer cells also reduced localization of GOF p53 on CCNA2 and CHK1 promoters and expression of cyclin A and CHK1 transcript and protein levels (Supplemental Figure 7). Taken together, these results show that GOF p53 mutants increase cyclin A and CHK1 expression at early $\mathrm{S}$ phase and that inactivation of this function abrogates cell proliferation, increase in origin firing, micronuclei formation, CHK1 activation, and chromatin association of $\gamma \mathrm{H} 2 \mathrm{AX}$. p53 Mutants with compromised tumor formation ability do not show localization on CCNA2 or CHK1 promoter, do not upregulate cyclin $A$ and CHK1 promoter to increase expression of respective transcripts, and fire fewer origins than GOF $p 53$ mutants. GOF p53 mutations often show allele specificity. To determine whether the ability of GOF p53 mutants to localize on the CCNA2 or CHK1 promoter, upregulate expression of cyclin A and CHK1, and increase origin firing relates to its tumor formation ability, we chose a naturally occurring p53 mutant, R267P, in human lung cancer cell line H1437. Tumor-formation ability of the H1437 cell line is not dependent on R267P (15). In addition, we tested a deletion mutant of GOF p53, D281G $\Delta 100-300$, that removes its central DNA binding domain (residues 100-300) and thus removes the site of hot spot mutation. ChIP analysis using p53-null H1299 lung cancer cells stably expressing empty vector, p53 D281G $\Delta 100-300$, R267P, R175H, R248W, or D281G as described above showed that, as in the case of H1299 cells stably expressing R273H (Figure 7), anti-p53 antibodies immunoprecipitated upstream promoter fragments of CCNA2 and CHK1 
from cells expressing p53 mutants R175H, R248W, or D281G, but not from cells expressing empty vector, p53 D281G $\Delta 100-300$, or R267P (Supplemental Figure 8, A and B).

Next, we analyzed the abilities of the p53 mutants to upregulate CCNA2 and CHK1 promoter activities. CCNA2 and CHK1 promoters containing the GOF p53-interaction sites as determined from our ChIP analysis (Figure 7) were inserted upstream of a luciferase gene in a reporter plasmid. The plasmids were introduced in p53-null H1299 cells along with the individual p53 mutants or vector plasmid, and luciferase activity was determined as reported earlier (16). Our data (Supplemental Figure 9, A-C) show that expression of GOF p53 mutants R175H, R248W, $\mathrm{R} 273 \mathrm{H}$, or D281G upregulated luciferase activity compared with empty vector or compared with the mutants p53 D281G $\Delta 100$ 300 or R267P. RT-QPCR of transcripts and immunoblot analysis of extracts prepared from partially synchronized H1299 cells stably expressing the p53 mutants showed that, consistent with the promoter activity, R175H, R248W, R273H, or D281G significantly increased cyclin A and CHK1 transcript and protein levels compared with empty vector or compared with the mutants p53 D281G 4100-300 or R267P (Supplemental Figure 9, D-H). Fiber analysis of replicating DNA from $\mathrm{H} 1299$ stable transfectants expressing the p53 mutants D281G $\Delta 100-300, \mathrm{R} 267 \mathrm{P}, \mathrm{R} 175 \mathrm{H}$, and $281 \mathrm{G}$ at early S phase showed that $\mathrm{H} 1299$ cells expressing R175H and $281 \mathrm{G}$ fired almost 3- to 4-fold more origins than cells expressing either D281G $\Delta 100-300$ or R267P (Supplemental Figure 9I). These results show that GOF p53 mutants localize on the CCNA2 and CHK1 promoter sequences, increase CCNA2 and CHK1 promoter activities and their expression, and increase origin firing at early $S$ phase, whereas p53 mutants deficient in GOF properties are inactive in these functions.

A small molecule inhibitor of CHK1 selectively reduces tumor size and growth of lung cancer cells with p53 mutation. If increased expression of CHK1 by GOF 553 protects replication fork progression (Figure 2) and thus accelerates genome duplication (Figure 3) and cell proliferation (Figure 5B), GOF p53-mediated cell proliferation should be selectively prevented by inhibition of CHK1 activity. To test this possibility, H1299 lung cancer cells stably expressing p53R273H or its transactivation-deficient mutant p53R273H TADI (Figure 5A) and H1299 cells stably transfected with vector control were treated with a CHK1 inhibitor, PF00477736, and the rate of cell proliferation was determined. The results of these experiments show that PF00477736 preferentially inhibited growth of cells expressing GOF p53R273H compared with p53R273H TADI or cells expressing vector control (Figure 9A and Supplemental Figure 7A). In a similar experiment, H1299 cells expressing p53 mutants D281G $\Delta 100-300$, R267P, R175H, and $281 G$ were treated with PF00477736 and cell number at 5 days was determined. Consistent with the previous finding, results (Figure 9B) of this experiment show that PF00477736 inhibited growth of H1299 cells expressing p53R175H or p53D281G more efficiently than cells expressing D281G $\Delta 100-300$ or R267P. Similarly, H1048 lung cancer cells (which naturally express p53R273C) stably expressing shRNA against p53 or control GFP were also treated with the CHK1 inhibitor, and rate of cell proliferation was determined by counting cell numbers in each case. Our data show that knockdown of GOF p53 remarkably reduced (2.5- to 3-fold) the sensitivity of the human lung cancer cell line H1O48 to the CHK1 inhibitor (Figure 9C). These observations indicate that the growth of H1299 cells expressing GOF p53 mutants is dependent on $C H K 1$ induction by GOF p53 and signify potential therapeutic efficiency of the CHK1 inhibitor in lung cancer cells expressing GOF p53 alleles.

To determine whether the CHK1 inhibitor preferentially targets cells that proliferate faster independently of GOF p53, we boosted cell proliferation by enriching growth media using $20 \%$ FBS and compared the efficiency of growth inhibition of mock-depleted and p53-depleted H1048 lung cancer cells or of vector-transfected H1299 cells with GOF p53 R273H-expressing H1299 cells in normal media by the CHK1 inhibitor PF00477736. Our data (Supplemental Figure 10) show that the enriched media increased cell growth in the presence or absence of CHK1 inhibitor. Although growth acceleration of p53-depleted H1048 cells was higher in the absence of CHK1 inhibitor compared with its presence, mock-depleted H1048 cells showed more sensitivity to CHK1 inhibitor in normal or enriched media (Supplemental Figure 10A). Enhanced proliferation of H1299 lung cancer cells (vector control) in enriched media did not increase efficiency of growth inhibition by CHK1 inhibitor (Supplemental Figure 10B). These data suggest that the efficiency of inhibition of cell growth by CHK1 inhibitor is not dependent on rate of proliferation alone.

If an increase in CHK1 expression, and therefore replication fork protection, renders cancer cells addicted to p53, inhibition of CHK1 should also reduce tumor growth. This possibility was examined using a mouse xenograft model. Subcutaneous tumors were allowed to form in immunodeficient $\mathrm{Nu} / \mathrm{Nu}$ mice $(\mathrm{Nu} / \mathrm{Nu}$ mice) by introducing $\mathrm{H} 1048$ cells stably expressing shGFP or shp53, and the effect of CHK1 inhibitor PF00477736 on tumor growth was examined. H1048 cells expressing shGFP efficiently formed tumors, while PF-00477736 treatment significantly reduced the size of the tumors (Figure 9, D-F). Knockdown of p53R273C with shp53 also efficiently reduced tumor size; however, PF-00477736 treatment did not reduce the size of the H1048shp53 tumors appreciably. Comparing the effect on H1048 shGFP and shp53 cells, PF-00477736 reduced size of tumors generated from H1048shGFP cells at 3.6- to 4-fold higher efficiency than that from H1048 shp53 cells (Supplemental Figure 11, B and C). These data indicate that tumors expressing GOF p53 are targeted efficiently and selectively by PF 00477736 in comparison with tumors not expressing GOF p53.

\section{Discussion}

Targeting GOF p53 mutants to prevent tumor growth or to treat established tumors has recently become an area of active investigation predicated on reversing the apparent addiction of tumor cells to the GOF properties of mutant p53 $(13,17,18)$. However, why or how growth of tumors or tumor cells becomes dependent on GOF p53 is not known.

The tumorigenic activity of GOF p53 and its ability to transcriptionally activate genes required for DNA replication (27, 50-52) drew the focus of this study on firing of DNA replication origins and progression of replication forks, the 2 crucial aspects of successful genome duplication. Consistent with the reports in literature (53-55), the presented ChIP-Seq and RNA-Seq analyses (Figure 6 
and Supplemental Figure 6) indicate that GOF p53 recognizes the promoter region of several genes involved in DNA replication and upregulates their expression, suggesting that the oncoprotein creates an intracellular environment conducive to accelerated genome duplication. Here, we present data to suggest what we believe is a novel oncogenic pathway (Figure 10, A and B), in which GOF p53dependent growth advantage of cancer cells lies in its ability to localize on the upstream regulatory sequences of CCNA2 and CHK1 (Figure 7 and Supplemental Figure 8), upregulating their expression (Figure 8 and Supplemental Figures 7 and 9). Since more origins are licensed than used (reviewed in refs. 36, 56), increase in cyclin A expression leads to firing of more origins of replication (Figure 1 and Supplemental Figure 9F), whereas increased CHK1 expression prevents their collapse (Figure 2), thus quickening genome duplication (Figure 3) and proliferation of cells with genetic abnormalities, as evidenced by micronuclei formation (Figure 4 and Figure $5 \mathrm{E}$ ). In contrast, due to their inability to upregulate cyclin A and CHK1 expression, p53-null cells or cells expressing GOF-deficient p53 mutants activate fewer origins than cells with GOF p53 (Figure 1) and face a vulnerability to collapse of replication forks (Figure 2), which is known to cause incomplete genome duplication, gross genome rearrangement, and cell death $(25,26)$.

Cell cycle progression is deregulated in the absence of WT p53, which normally induces expression of the CDK inhibitor p21 (57). Consistently, data presented here show that lung cells from either p53-null or p53R172H-KI mice enter S phase at similar times, with approximately $20 \%$ of the cells synthesizing DNA at early S phase while approximately $5 \%$ of lung cells from mice with WT p53 enter S phase at a similar time (Supplemental Figure 1). Thus, the presence of GOF $\mathrm{p} 53$ does not hasten time of S phase entry any sooner than loss of p53 (Supplemental Figure 1). However, expression analysis did reveal that at early $S$ phase, GOF p53 upregulates expression of cyclin A, required for origin activation, and CHK1, required for stabilization of replication forks (Figure 8 and Supplemental Figures 7-9) and, accordingly, increases the frequency of origin firing (Figure 1 and Supplemental Figure 9F) and prevents collapse of progressing replication forks (Figure 2), accelerating genome duplication and cell growth (Figure 3 and Figure 5B).

Oncogene-induced replication stress is thought to induce firing of dormant origins, which facilitates completion of genome duplication (58). However, the observed increase in origin firing by GOF p53 was concomitant to S phase entry of cells and was dependent on TADI of GOF p53, needed to upregulate cyclin A expression (Figure 5D). Since activation of CDK activates origins in replication factories $(59,60)$, GOF p53-induced cyclin A expression at early $S$ phase should activate origin firing in replication factories. Increases in the levels of p-CHK1 in GOF p53-expressing cells (Figure 2, A and B) may eventually fire dormant origins due to pausing of progressing replication forks at a later time point.

Our data also show that the increase in the frequency of origin firing by GOF p53 led to micronuclei formation in lung cells expressing GOF p53 (Figure 4). It has been reported that oncogene-induced increase in DNA replication induces fork reversal and generates unfinished replication intermediates (39). Although activation of CHK1 by GOF p53 prevents fork collapse, micronuclei formation in GOF p53-expressing cells may possibly be a consequence of mitotic processing of the intermediates generated by topological stress due to increase in origin firing as reported by Neelsen et al. (39). In support of this notion, reduced micronuclei formation was observed in the presence of a CDK2 inhibitor that is known to inhibit origin firing but allow fork progression (Table 1).

Supporting the central role of GOF p53-mediated transactivation of CHK1 in preventing collapse of replication forks, it was observed that a CHK1 inhibitor (Figure 2, G and H) or inhibition of CHK1 expression by transcriptional inactivation of GOF p53 leads to collapse of replication forks (Figure 5G). It is known that the collapse of replication forks leads to either cell death or gross genomic rearrangement due to incomplete genome duplication $(25,26)$. GOF p53-mediated fork stabilization, therefore, should allow the cells to evade these events detrimental to cell growth, although the cells enter S phase as early as p53-null cells. Accordingly, a CHK1 inhibitor preferentially inhibited proliferation of cells and growth of tumors expressing GOF p53 (Figure 9 and Supplemental Figure 11 ), while transactivation-deficient GOF p53 with substitution at the amino acid residues 22 and 23 was incapable of increasing origin firing, micronuclei formation, replication fork stabilization and induction of cell proliferation (Figure 5), and vulnerability to CHK1 inhibitor (Figure 9, A and B), implicating GOF p53 transactivation in regulating all these functions. Consistently, p53 mutants deficient in GOF property did not transactivate CCNA2 and CHK1 or increase origin firing and were not vulnerable to the CHK1 inhibitor (Supplemental Figure 9 and Figure 9B).

These results help explain the genetic instability and long latency period of tumorigenesis found in p53-null cells (61). Higher incidence of replication fork collapse and gross genome rearrangement in p53-null cells should lead to cell death, but also selection of genetically altered cells with survival advantage and tumorigenesis in the long run. GOF p53-driven increases in origin firing along with protection of replication forks should allow proliferation of cells with genetic abnormalities. These observations also explain dependence of tumor growth on GOF p53, lack of which would result in decreased origin firing and vulnerability to collapse of progressing replication forks, causing decelerated rates of genome duplication and lethal genome rearrangement. Our model for the role of GOF p53 in stimulating replication and simultaneously protecting replication forks (Figure 10) provides solid mechanistic justification for developing cancer cellselective therapeutics that target either GOF p53 transactivation function directly or target the protein products of GOF p53-transactivated genes. Such therapies, possibly in combination with other tumor type-specific targeted therapies, may lead to therapeutic gains in intractable cancers with high rates of GOF p53 mutation.

\section{Methods}

Plasmids, lentiviral vectors, and cell lines. Generation of plasmids, lentiviruses, and stable transfectants expressing GOF p53 or shRNA against GFP or p53 was carried out using pLKO.1 expression vector purchased from Open Biosystem following the supplier's protocols. Lung cells from mice were generated and cultured following standard protocols $(28,62)$. CCNA2 promoter was a gift from Toshio Nikaido (63). CHK1 promoter was constructed by isolating upstream promoter regions by PCR using primer sequences listed in Supplemental Table 1. H1299 and H1048 cell lines were obtained from ATCC. The VMRCLCD cell line was obtained from JCRB Cell Bank. 
Animals. p53-Null and p53R172H KI mice were generated by crossbreeding corresponding heterozygous $\left(p 53^{+/-}\right.$and $\mathrm{p} 53 \mathrm{R} 172 \mathrm{H} /$ WTp53, respectively) mice (gifts from Guillermina Lozanom, refs. 64, 65). Nude mice were obtained from Jackson Laboratory.

Chemicals and drugs. IdU and CldU were purchased from SigmaAldrich. PHA767491 (Tocris Bioscience) was used at a concentration of $10 \mu \mathrm{M}$ for indicated times $(46,47)$. CHK1 inhibitor PF-00477736 (Sigma-Aldrich) was used at a concentration of $50 \mathrm{nM}$ for 24 hours for treatment of cultured cells and $10 \mathrm{mg}$ per $\mathrm{kg}$ for treatment of tumors (35).

Antibodies. Antibodies used included anti-p53 (catalog sc-6243), CHK1 (catalog sc-56288), cyclin A (catalog sc-751), ERK-2 (catalog sc-154), and actin (catalog sc-47778) (Santa-Cruz Biotechnology Inc.); phospho CHK1 (catalog 2348s) (Cell Signaling Technology); $\gamma$-H2AX (catalog 05-636), and H3 (catalog 1953330) (Millipore). Antibodies were used per the manufacturer's protocol. Mitotic cells were identified by staining with $\alpha$-tubulin antibody (T9026) (Sigma-Aldrich). Antihuman p53 monoclonal antibody was a gift from David Lane (Agency for Science, Technology and Research, p53 Laboratory, Singapore). IdU was detected by mouse anti-bromodeoxyuridine (347580) from BD primary antibody and Alexa Fluor 594-conjugated rabbit anti-mouse and Alexa Fluor 594-conjugated goat anti-rabbit (Life Technologies) secondary and tertiary antibodies, respectively. CldU was detected by rat anti-bromodeoxyuridine (OBTO030G) primary antibody (Accurate) and Alexa Fluor 488-conjugated chicken anti-rat and Alexa Fluor 488conjugated goat anti-chicken (Life Technologies) secondary antibodies.

Detection of replicating nuclei. Replicating nuclei were detected following methods described earlier (28). Briefly, density-arrested cells were replated on coverslips and labeled with $40 \mu \mathrm{M}$ IdU for 20 minutes at desired times after replating. IdU was washed off using PBS. Cells were fixed with $3 \%$ paraformaldehyde solution. Fixed cells were treated for 5 minutes with $0.5 \%$ Triton X-100, followed by 1 hour treatment with $2.5 \mathrm{~N} \mathrm{HCl}$ to denature the DNA, and neutralized with 3 washes of $0.1 \mathrm{M}$ sodium borate. Cells were then washed with $0.1 \%$ Tween in PBS (wash buffer) and blocked in 2\% BSA in wash buffer for an hour. The cells were then sequentially incubated with primary and Alexa Fluor 594-conjugated secondary antibodies each for an hour in $1 \%$ BSA in wash buffer. The coverslips were washed and mounted on slides with ProLong Gold Antifade with DAPI (Life Technologies) and imaged using confocal microscopy (Zeiss LSM700).

Detection of replicating cells by flow cytometry. Flow cytometric analysis of replicating cells was performed using methods described earlier $(66,67)$. Cells were labeled with nucleotide analogue BrdU as described above. For flow cytometry, cells were fixed with $70 \%$ ethanol at $4^{\circ} \mathrm{C}$ overnight. Acid base treatment and immunostaining were conducted using the protocol described above using an Alexa Fluor 488-conjugated anti-BrdU antibody (BD Biosciences). DNA was stained with PI. The samples were analyzed using a BD FACScanto II flow cytometer using BD FACSDiva software.

Identification of DNA replication origin firing. DNA replication origin firing was determined by DNA fiber spreading analysis following published protocols $(28-30,68)$. Briefly, cells were pulse labeled sequentially with nucleotide analogues IdU $(40 \mu \mathrm{M})$ and CIdU (100 $\mu \mathrm{M})$ to track the replication pattern and directionality of fork movement. Cells were collected by trypsinization, and genomic DNA of approximately 600 cells was aligned on slides by fiber spreading as described earlier. Slides were then air dried, fixed in 3:1 methanol/ acetic acid, and dried overnight. After acid treatment $(2.5 \mathrm{~N} \mathrm{HCl}, 30$ minutes) and blocking (2\% BSA in PBS), DNA fibers on slides were immunostained with primary antibodies against IdU and CIdU followed by fluorescently labeled secondary and tertiary antibodies, washed, dried, and mounted in ProLong Gold Antifade (Life Technologies). Images were collected by confocal microscopy (Zeiss LSM700). Newly initiated single origins were detected as red track flanked on both sides by green track as explained in the legend of Figure 4A. Approximately 150 to 200 fibers from each sample were scored and analyzed using Image J software (NIH).

Cell cycle analysis and detection of mitotic nuclei. Cell cycle analysis was performed by ethanol fixation and PI staining at different time intervals after replating density-arrested cells and flow cytometry (BD FACSCanto II). Cell cycle analysis was performed using BD FACSDiva software. For identification of mitotic nuclei, density-arrested cells were replated on coverslips and fixed using 3\% paraformaldehyde. The cells were then treated with $0.1 \%$ Triton X-100 for 10 minutes, followed by PBS washes and 1 hour blocking in 5\% BSA. The primary antibody incubation was performed in the blocking buffer for 1 hour followed by PBS wash and 1 hour incubation with Alexa Fluor 488conjugated secondary antibody. Cells were briefly stained with DAPI solution, washed, mounted on slides using ProLong Gold Antifade, and imaged by confocal microscopy (Zeiss LSM700) at $\times 40$ magnification.

Detection of micronuclei. Cultured cells were plated on coverslips and fixed using 3\% paraformaldehyde, mounted on slides with ProLong Gold Antifade with DAPI, and imaged using confocal microscopy (Zeiss LSM700). Presence of micronuclei was examined for 500 randomly selected cells.

Detection of $\gamma H 2 A X$. Cultured cells were washed with PBS and harvested. The cell pellet was digested in lysis buffer (10 mM HEPES, pH 7.9, $1.5 \mathrm{mM} \mathrm{MgCl}_{2}, 10 \mathrm{mM} \mathrm{KCl}, 0.5 \mathrm{mM}$ DTT, $1.5 \mathrm{mM}$ PMSF) containing 0.2 $\mathrm{N} \mathrm{HCl}$ for 30 minutes. The supernatant was neutralized with phosphate buffer. Chromatin-bound $\gamma \mathrm{H} 2 \mathrm{AX}$ was detected by immunoblot analysis. For detection of $\gamma \mathrm{H} 2 \mathrm{AX}$ foci, cultured cells were plated on coverslips and fixed using $3 \%$ paraformaldehyde. The cells were then treated with $0.1 \%$ Triton X-100 for 10 minutes, followed by PBS washes and 1 hour blocking in 5\% BSA. The primary antibody incubation was performed in the blocking buffer for 1 hour followed by PBS wash and 1 hour incubation with Alexa Fluor 488-conjugated secondary antibody. Coverslips were mounted on slides using ProLong Gold Anitfade with DAPI and imaged by confocal microscopy (Zeiss LSM700) at $\times 40$ magnification; 250 cells were randomly examined for presence of $\gamma \mathrm{H} 2 \mathrm{AX}$.

Immunoblot analysis and quantification. Immunoblot analysis was performed following standard techniques. Antibodies are described above. Quantitative comparisons were performed using Quantity One 4.6.2 software (Bio-Rad).

RNA extraction and sequencing. Total RNA was isolated from exponentially growing cultured cell lines using TRIzol reagent (Life Technologies) following the supplier's protocols. For RNA-Seq, $4 \mu \mathrm{g}$ of total RNA was processed and sequenced at Donnelly Sequencing Center at the University of Toronto (Toronto, Canada). RNA samples were run on the Illumina HiSeq 2500 System, and individual fastq files were used for analysis. Sequences were aligned to the human genome (HG19) and analyzed using DNASTAR ArrayStar (version 12) software. All RNA-Seq experiments were normalized by assigned reads per kilobase of template per million (RPKM) mapped reads. RNA-sequencing information can be found in the ArrayExpress database (accession umber: E-MTAB-5652). For more information, see Supplemental Table 2. 
ChIP analysis and sequencing. ChIP and ChIP-Seq were performed using standard methods (69). Briefly, exponentially growing H1299 cells expressing either empty vector or the p53 mutant p53R273H were fixed with formaldehyde, extracted, and immunoprecipitated with antibodies against p53 (DO1 and FL-393, Santa Cruz Biotechnology Inc.). DNA fragments were eluted from immunoprecipitates, reverse crosslinked, and purified. Purified DNA (150 ng) was sequenced at the Donnelly Sequencing Center at the University of Toronto. ChIP samples were run on the Illumina HiSeq 2500 System, and individual fastq files were used for analysis. Sequences were aligned to the human genome (HG19) and analyzed using DNASTAR ArrayStar (version 12) software. Peaks were visualized using SeqMan Pro software (DNASTAR). ChIP-Seq experiments were normalized by reads assigned per million mapped reads (RPM). Genome sequences that showed binding of p53 in p53-R273H-expressing cells but no binding in vector cells or where there was greater than 1.5 -fold higher binding in the p53$\mathrm{R} 273 \mathrm{H}$ cells were chosen for further analysis. Gene lists for RNA-Seq and ChIP-Seq were compared to determine which genes were upregulated by mutant $\mathrm{p} 53$ and which also have $\mathrm{p} 53$ binding to the promoter.

For ChIP analysis, DNA samples were suspended in water and the presence of cyclin A and CHK1 promoter fragments were analyzed by 3 contiguous sets of primers as listed in Supplemental Table 1.

Generation of $c D N A$ and quantitative PCR. RNA extraction, cDNA preparation, and quantitative PCR (QPCR) were performed following standard protocols (27). cDNA was synthesized using the Thermoscript Reverse Transcription-PCR system (Life Technologies). QPCR was carried out using a LightCycler system (Roche). Primers (Supplemental Table 1) were designed using OLIGO 5 software (Molecular Biology Insights) and were synthesized by Integrated DNA Technologies. Reactions were performed in duplicate utilizing SYBR green dye, which exhibits a higher fluorescence upon binding of double-stranded DNA.

Analysis of CCNA2 and CHK1 promoter activity. $\mathrm{H} 1299$ cells were transfected with $200 \mathrm{ng}$ of luciferase reporter plasmid (pGL3), with luciferase reporter under the control of CCNA2 (63) or CHK1 promoter sequences or empty pGL3 basic vector (Promega), and $1 \mu \mathrm{g}$ of the indicated p53 expression plasmid using Lipofectamine 2000 (Invitrogen) following the manufacturer's instructions. Cell lysates were prepared 48 hours after transfection, and luciferase activity was determined. Luciferase analysis was carried out using the luciferase assay system (E1500) and instructions from Promega. Experiments were performed in triplicate as described before (16).

Xenograft studies. $\mathrm{Nu} / \mathrm{Nu}$ mice were used for the tumorigenicity studies. Eight-week-old mice were injected with $1 \times 10^{7}$ cells subcutane- ously in both flanks and measured periodically following published protocols (70). The H1048 cell line expressing shRNA against p53 or GFP was used. Tumor size was assessed at day 10, and mice were equally distributed into 2 groups to receive daily $10 \mathrm{mg} / \mathrm{kg}$ of PF-0047773 dissolved in 2\% DMSO, 40\% PEG, or vehicle by intraperitoneal injection.

Statistics. Unless otherwise specified, all experiments were performed in triplicate and representative data are shown. The presence of bidirectional origins as well as collapsed forks detected by sequential labeling of replicating DNA in different samples in each experiment set were compared using Student's $t$ test. Percentages of mitotic cells between different samples were compared using Student's $t$ test. Micronuclei per cell for different samples were evaluated using the $\chi^{2}$ test. QPCR reactions were performed in duplicate and repeated in 3 independent experiments. All immunoblots were repeated 3 times, and representative data are shown. Unless otherwise specified, for all the experiments, a $P$ value of less than 0.05 was considered significant.

Study approval. All animal studies were approved by the Institutional Animal Care and Use Committee (IACUC) at the Virginia Commonwealth University.

\section{Author contributions}

SS generated data to show cell cycle-dependent expression of cyclin A and CHK1, micronuclei and $\gamma \mathrm{H} 2 \mathrm{AX}$ foci formation, and replication fork collapse. SS and CAV generated mouse constructs and cultured primary cells. SD and CAV generated and analyzed RNA-Seq and ChIP-Seq data. RAF contributed in determining frequency of origin firing. SRG and SD contributed to experimental design. SPD conceived and designed experiments, supervised the work, and wrote the manuscript. All authors contributed to data analysis and preparation of the manuscript.

\section{Acknowledgments}

The authors wish to thank G. Lozano for her generous gift of p53R172H/WTp53 and $p 53^{+/-}$mice. The study was supported by institutional pilot projects to SPD and SD and NCI R01-CA107532 to SRG.

Address correspondence to: Swati Palit Deb, Sumitra Deb, or Steven R. Grossman, Goodwin Research Laboratories, 401 College Street, Richmond, Virginia 23298, USA. Phone: 804.828.9541; E-mail: Swati.Deb@vcuhealth.org (S.P.Deb).Phone: 804.827.1375; E-mail: sumitradeb2009@gmail.com (S. Deb). Phone: 804.828.5662; E-mail: steven.grossman@vcuhealth.org (S.R. Grossman).
1. Chang YL, Wu CT, Shih JY, Lee YC. Comparison of 553 and epidermal growth factor receptor gene status between primary tumors and lymph node metastases in non-small cell lung cancers. Ann Surg Oncol. 2011;18(2):543-550.

2. Harris CC. p53 tumor suppressor gene: from the basic research laboratory to the clinic--an abridged historical perspective. Carcinogenesis. 1996;17(6):1187-1198.

3. Olivier M, Hollstein M, Hainaut P. TP53 mutations in human cancers: origins, consequences, and clinical use. Cold Spring Harb Perspect Biol. 2010;2(1):a001008.
4. Bronte G, et al. Driver mutations and differential sensitivity to targeted therapies: a new approach to the treatment of lung adenocarcinoma. Cancer Treat Rev. 2010;36 Suppl 3:S21-S29.

5. Soussi T, Wiman KG. TP53: an oncogene in disguise. Cell Death Differ. 2015;22(8):1239-1249.

6. Olive KP, et al. Mutant p53 gain of function in two mouse models of Li-Fraumeni syndrome. Cell. 2004;119(6):847-860.

7. Krepulat F, Löhler J, Heinlein C, Hermannstädter A, Tolstonog GV, Deppert W. Epigenetic mechanisms affect mutant p53 transgene expression in WAP-mutp53 transgenic mice. Oncogene.
2005;24(29):4645-4659.

8. Ghebranious N, Sell S. The mouse equivalent of the human p53ser249 mutation p53ser246 enhances aflatoxin hepatocarcinogenesis in hepatitis B surface antigen transgenic and p53 heterozygous null mice. Hepatology. 1998;27(4):967-973.

9. Zheng S, El-Naggar AK, Kim ES, Kurie JM, Lozano G. A genetic mouse model for metastatic lung cancer with gender differences in survival. Oncogene. 2007;26(48):6896-6904.

10. Hanel W, Marchenko N, Xu S, Yu SX, Weng W, Moll U. Two hot spot mutant p53 mouse models display differential gain of function in tumori- 
genesis. Cell Death Differ. 2013;20(7):898-909.

11. Freed-Pastor WA, Prives C. Mutant p53: one name, many proteins. Genes Dev. 2012;26(12):1268-1286.

12. Oren M, Rotter V. Mutant p53 gain-of-function in cancer. Cold Spring Harb Perspect Biol. 2010;2(2):a001107.

13. Vaughan C, Pearsall I, Yeudall A, Deb SP, Deb S. p53: its mutations and their impact on transcription. Subcell Biochem. 2014;85:71-90.

14. Zhou G, et al. Gain-of-function mutant p53 promotes cell growth and cancer cell metabolism via inhibition of AMPK activation. Mol Cell. 2014;54(6):960-974.

15. Vaughan CA, et al. Allele specific gain-offunction activity of p53 mutants in lung cancer cells. Biochem Biophys Res Commun. 2012;428(1):6-10

16. Vaughan CA, et al. p53 mutants induce transcription of NF-kB2 in H1299 cells through CBP and STAT binding on the NF- $\mathrm{kB} 2$ promoter and gain of function activity. Arch Biochem Biophys. 2012;518(1):79-88.

17. Alexandrova EM, et al. Improving survival by exploiting tumour dependence on stabilized mutant p 53 for treatment. Nature. 2015;523(7560):352-356.

18. Pagliarini R, Shao W, Sellers WR. Oncogene addiction: pathways of therapeutic response, resistance, and road maps toward a cure. $E M B O$ Rep. 2015;16(3):280-296.

19. Chibazakura T, Kamachi K, Ohara M, Tane S, Yoshikawa H, Roberts JM. Cyclin A promotes S-phase entry via interaction with the replication licensing factor Mcm7. Mol Cell Biol. 2011;31(2):248-255.

20. Coverley D, Laman H, Laskey RA. Distinct roles for cyclins $\mathrm{E}$ and $\mathrm{A}$ during DNA replication complex assembly and activation. Nat Cell Biol. 2002;4(7):523-528.

21. Girard F, Strausfeld U, Fernandez A, Lamb $\mathrm{NJ}$. Cyclin A is required for the onset of DNA replication in mammalian fibroblasts. Cell. 1991;67(6):1169-1179.

22. Lecona E, Fernández-Capetillo O. Replication stress and cancer: it takes two to tango. Exp Cell Res. 2014;329(1):26-34.

23. Lopes M, et al. The DNA replication checkpoint response stabilizes stalled replication forks. Nature. 2001;412(6846):557-561.

24. Magdalou I, Lopez BS, Pasero P, Lambert SA. The causes of replication stress and their consequences on genome stability and cell fate. Semin Cell Dev Biol. 2014;30:154-164.

25. Carr AM, Paek AL, Weinert T. DNA replication: failures and inverted fusions. Semin Cell Dev Biol. 2011;22(8):866-874

26. Cortez D. Preventing replication fork collapse to maintain genome integrity. DNA Repair (Amst). 2015;32:149-157.

27. Scian MJ, et al. Tumor-derived p 53 mutants induce oncogenesis by transactivating growth-promoting genes. Oncogene. 2004;23(25):4430-4443.

28. Frum RA, et al. The human oncoprotein MDM2 induces replication stress eliciting early intraS-phase checkpoint response and inhibition of DNA replication origin firing. Nucleic Acids Res. 2014;42(2):926-940.

29. Frum RA, Deb S, Deb SP. Use of the DNA fiber spreading technique to detect the effects of mutant 553 on DNA replication. Methods Mol Biol. 2013;962:147-155.

30. Dorn ES, Chastain PD, Hall JR, Cook JG. Analysis of re-replication from deregulated origin licensing by DNA fiber spreading. Nucleic Acids Res. 2009;37(1):60-69.

31. Di Micco R, et al. Oncogene-induced senescence is a DNA damage response triggered by DNA hyper-replication. Nature. 2006;444(7119):638-642.

32. Herold S, Herkert B, Eilers M. Facilitating replication under stress: an oncogenic function of MYC Nat Rev Cancer. 2009;9(6):441-444

33. Gagou ME, Zuazua-Villar P, Meuth M. Enhanced H2AX phosphorylation, DNA replication fork arrest, and cell death in the absence of Chk1. Mol Biol Cell.2010;21(5):739-752.

34. Zuazua-Villar P, Rodriguez R, Gagou ME, Eyers PA, Meuth M. DNA replication stress in CHK1-depleted tumour cells triggers premature (S-phase) mitosis through inappropriate activation of Aurora kinase B. Cell Death Dis. 2014; $5:$ e1253.

35. Sarmento LM, et al. CHK1 overexpression in T-cell acute lymphoblastic leukemia is essential for proliferation and survival by preventing excessive replication stress. Oncogene. 2015;34(23):2978-2990.

36. Zeman MK, Cimprich KA. Causes and consequences of replication stress. Nat Cell Biol. 2014;16(1):2-9.

37. Bartkova J, et al. Oncogene-induced senescence is part of the tumorigenesis barrier imposed by DNA damage checkpoints. Nature. 2006;444(7119):633-637.

38. Blow JJ, Gillespie PJ. Replication licensing and cancer--a fatal entanglement? Nat Rev Cancer. 2008;8(10):799-806.

39. Neelsen KJ, Zanini IM, Herrador R, Lopes M. Oncogenes induce genotoxic stress by mitotic processing of unusual replication intermediates. J Cell Biol. 2013;200(6):699-708.

40. Neelsen KJ, et al. Deregulated origin licensing leads to chromosomal breaks by rereplication of a gapped DNA template. Genes Dev. 2013;27(23):2537-2542.

41. Battershill JM, Burnett K, Bull S. Factors affecting the incidence of genotoxicity biomarkers in peripheral blood lymphocytes: impact on design of biomonitoring studies. Mutagenesis. 2008;23(6):423-437.

42. Kirsch-Volders $M$, et al. The in vitro MN assay in 2011: origin and fate, biological significance, protocols, high throughput methodologies and toxicological relevance. Arch Toxicol. 2011;85(8):873-899.

43. Luzhna L, Kathiria P, Kovalchuk O. Micronuclei in genotoxicity assessment: from genetics to epigenetics and beyond. Front Genet. 2013;4:131.

44. Bell SP, Dutta A. DNA replication in eukaryotic cells. Annu Rev Biochem. 2002;71:333-374.

45. Nakanishi M, Katsuno Y, Niida H, Murakami H, Shimada M. Chk1-cyclin A/Cdk1 axis regulates origin firing programs in mammals. Chromosome Res. 2010;18(1):103-113.

46. Jones RM, et al. Increased replication initiation and conflicts with transcription underlie
Cyclin E-induced replication stress. Oncogene. 2013;32(32):3744-3753.

47. Montagnoli A, et al. A Cdc7 kinase inhibitor restricts initiation of DNA replication and has antitumor activity. Nat Chem Biol. 2008;4(6):357-365.

48. Beckerman R, Prives C. Transcriptional regulation by p53. Cold Spring Harb Perspect Biol. 2010;2(8):a000935.

49. Muller PA, Vousden KH. p53 mutations in cancer. Nat Cell Biol. 2013;15(1):2-8.

50. Lányi A, Deb D, Seymour RC, Ludes-Meyers JH, Subler MA, Deb S. 'Gain of function' phenotype of tumor-derived mutant $\mathrm{p} 53$ requires the oligomerization/nonsequence-specific nucleic acid-binding domain. Oncogene. 1998;16(24):3169-3176.

51. Lin J, Teresky AK, Levine AJ. Two critical hydrophobic amino acids in the $\mathrm{N}$-terminal domain of the $\mathrm{p} 53$ protein are required for the gain of function phenotypes of human p53 mutants. Oncogene. 1995;10(12):2387-2390.

52. Scian MJ, et al. Modulation of gene expression by tumor-derived p 53 mutants. Cancer Res. 2004;64(20):7447-7454.

53. Di Agostino $S$, et al. YAP enhances the proproliferative transcriptional activity of mutant p53 proteins. EMBO Rep. 2016;17(2):188-201.

54. Zhu J, et al. Gain-of-function p53 mutants co-opt chromatin pathways to drive cancer growth. Nature. 2015;525(7568):206-211.

55. Di Agostino S, et al. Gain of function of mutant p53: the mutant p53/NF-Y protein complex reveals an aberrant transcriptional mechanism of cell cycle regulation. Cancer Cell. 2006;10(3):191-202.

56. Hills SA, Diffley JF. DNA replication and oncogene-induced replicative stress. Curr Biol. 2014;24(10):R435-R444

57. Warfel NA, El-Deiry WS. p21WAF1 and tumourigenesis: 20 years after. Curr Opin Oncol. 2013;25(1):52-58.

58. Alver RC, Chadha GS, Blow JJ. The contribution of dormant origins to genome stability: from cell biology to human genetics. DNA Repair (Amst). 2014;19:182-189.

59. Thomson AM, Gillespie PJ, Blow JJ. Replication factory activation can be decoupled from the replication timing program by modulating $\mathrm{Cdk}$ levels. J Cell Biol. 2010;188(2):209-221.

60. Ge XQ, Blow JJ. Chk1 inhibits replication factory activation but allows dormant origin firing in existing factories. J Cell Biol. 2010;191(7):1285-1297.

61. Donehower LA, et al. Mice deficient for p53 are developmentally normal but susceptible to spontaneous tumours. Nature. 1992;356(6366):215-221.

62. Singh S, Ramamoorthy M, Vaughan C, Yeudall WA, Deb S, Palit Deb S. Human oncoprotein MDM2 activates the Akt signaling pathway through an interaction with the repressor element-1 silencing transcription factor conferring a survival advantage to cancer cells. Cell Death Differ. 2013;20(4):558-566.

63. Yamamoto M, et al. Effect of tumor suppressors on cell cycle-regulatory genes: RB suppresses $\mathrm{p} 34 \mathrm{cdc} 2$ expression and normal p53 suppresses cyclin A expression. Exp Cell Res. 1994;210(1):94-101. 
64. Montes de Oca Luna R, Wagner DS, Lozano G. Rescue of early embryonic lethality in $\mathrm{mdm} 2$-deficient mice by deletion of p53. Nature. 1995;378(6553):203-206.

65. Terzian T, et al. The inherent instability of mutant p53 is alleviated by Mdm2 or p16INK4a loss. Genes Dev. 2008;22(10):1337-1344.

66. Brown DR, Thomas CA, Deb SP. The human oncoprotein MDM2 arrests the cell cycle: elimi- nation of its cell-cycle-inhibitory function induces tumorigenesis. EMBO J. 1998;17(9):2513-2525.

67. Frum R, Deb SP. Flow cytometric analysis of MDM2-mediated growth arrest. Methods Mol Biol. 2003;234:257-267.

68. Frum RA, Chastain PD, Qu P, Cohen SM, Kaufman DG. DNA replication in early $S$ phase pauses near newly activated origins. Cell Cycle. 2008;7(10):1440-1448.
69. Robertson G, et al. Genome-wide profiles of STAT1 DNA association using chromatin immunoprecipitation and massively parallel sequencing. Nat Methods. 2007;4(8):651-657.

70. Sarmento LM, et al. CHK1 overexpression in T-cell acute lymphoblastic leukemia is essential for proliferation and survival by preventing excessive replication stress. Oncogene. 2015;34(23):2978-2990. 